\title{
Modulation of Inhibitory Synaptic Potentials in the Piriform Cortex
}

\author{
MADHVI M. PATIL AND MICHAEL E. HASSELMO \\ Department of Psychology, Boston University, Boston 02215; and Department of Psychology and Program in Neuroscience, \\ Harvard University, Cambridge, Massachusetts 02138
}

Patil, Madhvi M. and Michael E. Hasselmo. Modulation of inhibitory synaptic potentials in the piriform cortex. J. Neurophysiol. 81: 2103-2118, 1999. Intracellular recordings from pyramidal neurons in brain slice preparations of the piriform cortex were used to test results from a computational model about the effects of cholinergic agonists on inhibitory synaptic potentials induced by stimulation of afferent fibers in layer Ia and association/intrinsic fibers in layer Ib. A simple model of piriform cortex as an associative memory was used to analyze how suppression of inhibitory synaptic transmission influenced performance of the network. Levels of suppression of excitatory synaptic transmission were set at levels determined in previous experimental work. Levels of suppression of inhibitory synaptic transmission were then systematically varied within the model. This modeling work demonstrated that suppression of inhibitory synaptic transmission in layer Ib should be stronger than suppression of inhibitory synaptic transmission in layer Ia to keep activity levels high enough for effective storage. Experimental data showed that perfusion of the cholinergic agonist carbachol caused a significant suppression of inhibitory postsynaptic potentials (IPSPs) in the pyramidal neurons that were induced by stimulation of layer $\mathrm{Ib}$, with a weaker effect on IPSPs induced by stimulation of layer Ia. As previously described, carbachol also selectively suppressed excitatory postsynaptic potentials (EPSPs) elicited by intrinsic but not afferent fiber stimulation. The decrease in amplitude of IPSPs induced by layer Ib stimulation did not appear to be directly related to the decrease in EPSP amplitude induced by layer Ib stimulation. The stimulation necessary to induce neuronal firing with layer Ia stimulation was reduced in the presence of carbachol, whereas that necessary to induce neuronal firing with layer Ib stimulation was increased, despite the depolarization of resting membrane potential. Thus physiological data on cholinergic modulation of inhibitory synaptic potentials in the piriform cortex is compatible with the functional requirements determined from computational models of piriform cortex associative memory function.

\section{N T R O D U C T I O N}

The dynamical interactions of cortical neurons can be altered by a range of modulatory substances, including ACh (for review see Hasselmo 1995). ACh has been shown to suppress excitatory synaptic transmission in the piriform cortex (Hasselmo and Bower 1992; Linster et al. 1999; Williams and Constanti 1988), in the hippocampus (Hasselmo et al. 1995; Valentino and Dingledine 1981; Yamamoto and Kawai 1967), and in the neocortex (Hasselmo and Cekic 1996). This effect shows laminar selectivity, with stronger suppression of excitatory synaptic transmission at synapses between pyramidal cells within a region and weaker suppression at synapses arising from other areas (Hasselmo and Bower 1992; Hasselmo and Schnell 1994). This selectivity may be of particular functional relevance, as demonstrated in computational models of cortical function (Hasselmo and Bower 1993; Hasselmo et al. 1992). In the presence of cholinergic modulation, suppression of intrinsic synaptic transmission allows afferent sensory input to more strongly drive the activity within cortical network models, setting appropriate dynamics for attention to the external environment and storage of new information. Cholinergic modulation has been shown to enhance long-term potentiation of synaptic potentials in the piriform cortex (Hasselmo and Barkai 1995; Patil et al. 1998), further setting the appropriate dynamics for storage of new information.

Cholinergic agonists have also been shown to suppress evoked inhibitory synaptic potentials (Haas 1982; Muller and Misgeld 1986; Pitler and Alger 1992; Valentino and Dingledine 1981). This has been shown in brain slice preparations of the hippocampal formation and cultures of neocortex but was not previously analyzed in piriform cortex. Computational models of cortical function can be used to analyze the functional significance of the cholinergic suppression of inhibitory synaptic transmission. As described here, computational models of associative memory function in the piriform cortex generated the prediction that cholinergic modulation should cause greater suppression of inhibitory synaptic potentials elicited by stimulation of intrinsic and association fibers in layer Ib than inhibitory synaptic potentials elicited by stimulation of afferent fibers in layer Ia. This prediction was tested with physiological recording of inhibitory synaptic potentials in brain slice preparations of the piriform cortex. Experimental work investigated whether the cholinergic modulation of inhibitory synaptic potentials observed in hippocampus appears in the piriform cortex as well and whether this modulation shows the laminar selectivity suggested by the computational modeling work.

\section{METHODS}

\section{Mathematical analysis of piriform cortex modeling}

ACh simultaneously alters a number of different parameters of cortical neurons. A simple mathematical model of cortical circuits helps in understanding the interaction of these modulatory effects. In this model, we assumed that the maximum steady-state activity of excitatory neurons should remain about the same during changes in ACh levels. The pattern of responsiveness of individual neurons does change during cholinergic modulation (Metherate et al. 1990; Sillito and Kemp 1983), but physiologically realistic changes in cholinergic modulation do not cause a dramatic change in overall activity such as a total absence of activity. On a functional level, modulatory changes would be considerably more useful if they would change the pattern of neuronal response rather than completely shut off the network or cause it to become overactive. Thus we assume that the level of activity should remain within a stable range. Here we evaluated how much modulatory change in inhibitory synaptic transmission would be necessary to offset the modulation of excitatory synaptic transmission previously described in the piriform cortex (Hasselmo and Bower 1992; Linster et al. 1999).

This model focuses on the interaction of populations of excitatory 
units and a population of inhibitory units mediating feedforward and feedback inhibition. This representation has a considerable advantage over other neural network models in which excitatory units and inhibitory units are not represented separately (Amit 1988). Dynamics of the mathematical representation used here were first studied by Wilson and Cowan $(1972,1973)$. This type of representation was used to study the dynamics of cortical networks including piriform cortex (Hasselmo and Linster 1998a,b; Hasselmo et al. 1997), hippocampus (Hasselmo et al. 1995; Tsodyks et al. 1997), somatosensory cortex (Pinto et al. 1996), and visual cortex (Hansel and Sompolinsky 1998). These models leave out many of the details incorporated in compartmental biophysical simulations (e.g., Barkai and Hasselmo 1994; Hasselmo and Barkai 1995), such as the Hodgkin-Huxley current underlying spike generation, and the passive properties of dendritic trees. Thus they are somewhat less constrained with regard to the intrinsic properties of individual neurons. However, the network dynamics of these simplified representations show many qualitative features in common with spiking network models, including attractor dynamics (Fransen and Lansner 1995; Hansel and Sompolinsky 1998; Pinto et al. 1996), and the results of the analysis presented here should apply to network dynamics in a biophysical simulation.

In these models the firing rate of a population of neurons is simplified into a continuous firing rate variable, which depends on the average membrane potential of the population. The firing of a spike in an individual neuron is an all-or-nothing phenomenon, but the spiking rate within a population can be seen as a continuous variable, which is zero when the average membrane potential of the population is well below threshold, small when the average membrane potential is just above threshold, and large when the average membrane depolarization is large. In the computational simulations described in the next section, we split the population of excitatory neurons into separate populations representing components of different odor patterns while simulating just one population of inhibitory neurons. For the mathematical analysis described in this section, we focus on the average membrane potential (represented by the variable $a$ ) of one subpopulation of excitatory neurons and the average membrane potential (represented by the variable $h$ ) of the subpopulation of inhibitory neurons that interacts with these excitatory neurons. (These averages correspond to the membrane potential determined by synaptic input and exclude the membrane potential during generation of spikes).

Changes in the average membrane potential of the population of excitatory and inhibitory neurons are described by the following equations

$$
\begin{gathered}
\mathrm{da} / \mathrm{dt}=A-\eta a+W \phi[a-\theta]_{+}-H \phi[h-\theta]_{+} \\
\mathrm{dh} / \mathrm{dt}=A^{\prime}-\eta^{\prime} h+W^{\prime} \phi[a-\theta]_{+}
\end{gathered}
$$

These equations show the change in average membrane potential $(a)$ of the excitatory population and average membrane potential $(h)$ of the inhibitory population (in units that correspond to $\mathrm{mV}$ from resting potential). The constant $\eta$ multiplied by $a$ represents the passive decay of membrane potential proportional to the difference from resting potential. This constant has units of milliseconds and is the inverse of the average membrane time constant. In biophysical simulations, the membrane time constant is calculated as the product of membrane resistance and capacitance. The summed firing rate of the excitatory population (in spikes/ms) is computed by a threshold linear function $[a-\theta]_{+}$of average membrane potential scaled by a summation factor $\phi$. We use a sum of firing rates, not the average firing rate (which would be $<1$ spike/ms), because each neuron receives a large number of synaptic inputs that are simplified to one input here. These firing rates are zero when membrane potential is $\angle \theta(\theta=8 \mathrm{mV}$ in the accompanying simulations). Firing rates take the value $\phi(a-\theta)$ for values $>\theta$. Piriform cortex pyramidal cells do not normally fire above $100 \mathrm{~Hz}$, and within this range of firing their $f-I$ curve has a threshold linear shape (Barkai and Hasselmo 1994). For the analysis presented here we set $\phi=1$; therefore it will not appear in any subsequent equations. The same type of threshold linear function is used for computing the summed firing rate of the inhibitory population. The constant $A$ represents the afferent input to a population of neurons during a period of time. This constant represents both the summed firing rate across a population of mitral cells in the olfactory bulb in spikes/ms as well as the process of synaptic transmission at afferent fiber synapses in layer Ia of piriform cortex (in $\mathrm{mV} / \mathrm{spike}$ ). The product of these values has the necessary value of spikes/ms $\times \mathrm{mV}$ / spike $=\mathrm{mV} / \mathrm{ms}$.

In these equations, $W$ represents the average strength of excitatory synapses arising from cortical pyramidal cells and synapsing on other excitatory neurons. If neuronal output is in spikes/ms, then synaptic strength reflects the change in membrane voltage per spike $(\mathrm{mV} /$ spike) because of the membrane conductance change caused by synaptic transmission. $H$ represents the average strength of inhibitory synapses arising from cortical inhibitory interneurons and synapsing on pyramidal cells. $W^{\prime}$ represents the average strength of excitatory synapses arising from cortical pyramidal cells and synapsing on inhibitory interneurons. To keep the equations simpler, we left out inhibitory synapses on inhibitory interneurons, which were included in previous work. The simplified system is summarized in Fig. $1 A$.

The equilibrium states of networks of this type were evaluated in a previous article (Hasselmo et al. 1995). Here we use those equilibrium states to investigate the relationship between modulation of excitatory synaptic transmission and modulation of inhibitory synaptic transmission. Previous experimental work has shown the magnitude of cholinergic suppression of excitatory synaptic potentials in the afferent and intrinsic fiber layers of the piriform cortex (Hasselmo and Bower 1992). The cholinergic suppression of excitatory synaptic transmission was modeled in the equations by rescaling the excitatory intrinsic connections $W$ proportional to a unitless suppression variable $c_{w}$ and scaling the excitatory connections from pyramidal cells to interneurons $W^{\prime}$ proportional to a suppression variable $c_{w^{\prime}}$ Given these values, we evaluated how feedforward and feedback inhibition should change to keep the equilibrium activity of the network in the same range of values and for the network to remain stable. Modulation of feedforward inhibition $H_{f f}$ was represented by the variable $c_{f f}$, and modulation of feedback inhibition $H$ was represented by the variable $c_{f b}$ (all suppression variables range between 0 and 1.0). As described in the RESULTS, modulation of excitatory synaptic transmission of the sort described previously (Hasselmo and Bower 1992) was more effectively offset by modulation of feedback inhibitory parameters than by modulation of feedforward inhibition.

\section{Computational modeling}

The functional significance of different levels of the cholinergic modulation of inhibitory synaptic potentials was analyzed in a simplified computational model of the piriform cortex, showing that selective cholinergic suppression of feedback but not feedforward inhibition is necessary for effective function. This computational model used the same general functional framework as the mathematical analysis described previously, but instead of a single subpopulation of excitatory neurons the computational model split the population of excitatory neurons into several excitatory units representing separate subpopulations of excitatory neurons responding differentially to different components of different odor patterns. Many aspects of cholinergic modulation were analyzed in previous compartmental biophysical simulations of the piriform cortex with spiking neurons (Barkai et al. 1994; Hasselmo and Barkai 1995), but this computational model used the simplified representation of average firing rate described previously (Hasselmo et al. 1995; Pinto et al. 1996; Wilson and Cowan 1972). This simplified representation was used previously to analyze cholinergic modulation in region CA3 of the hippocampus. In fact, that previous simulation used selective cholinergic suppression of feedback but not feedforward inhibition, although the full range of parameter values was not explored. In the computational simulations described in the next section, we split the population of 
B
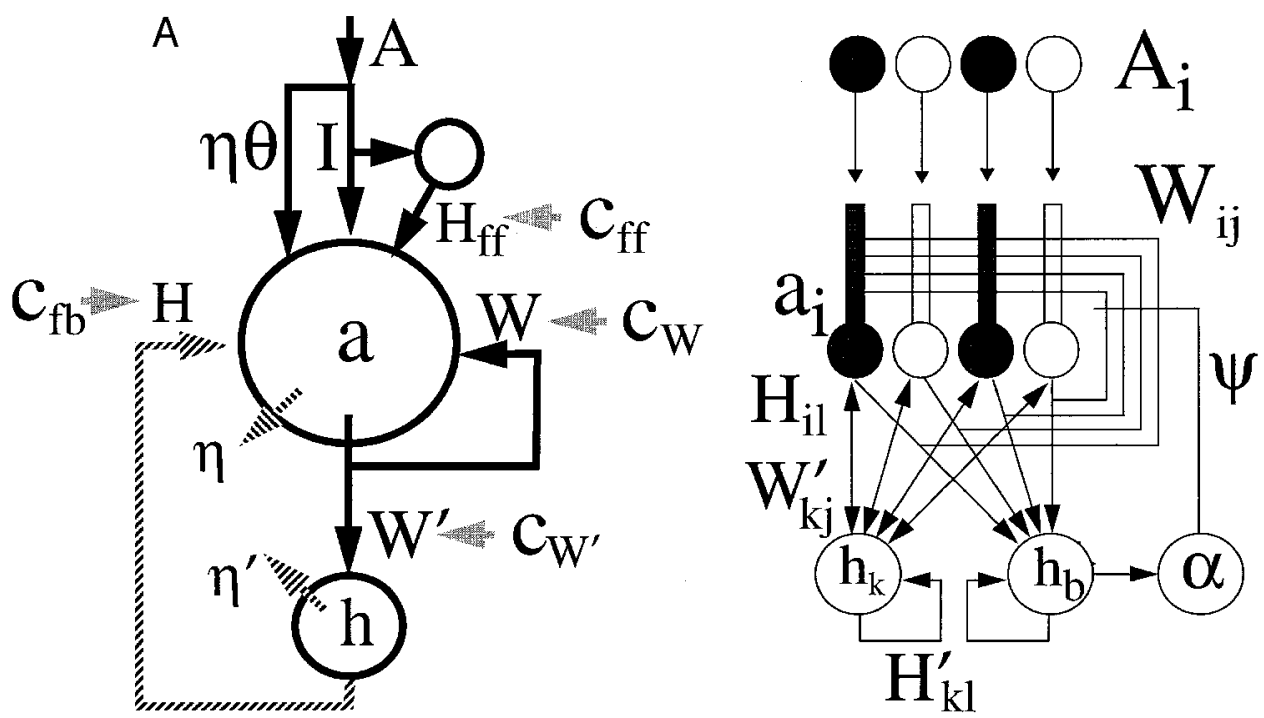

FIG. 1. A: simplified network for mathematical analysis. a: average membrane potential of a subpopulation of excitatory neurons; h: average membrane potential of a population of inhibitory neurons. $A=$ afferent input from olfactory bulb mitral cells passing through layer Ia synapses. This is subdivided into subthreshold input $(\eta \times \theta)$ and suprathreshold input (I) minus feedforward inhibition $\left(H_{f f}\right) \cdot c_{f f}=$ cholinergic modulation of feedforward inhibition. $W=$ feedback excitatory connections between pyramidal cells in the network, scaled by cholinergic modulation $c_{w} . W^{\prime}=$ excitatory connections to inhibitory interneuron, scaled by cholinergic modulation $c_{w}, H_{\mathrm{int}}=$ inhibitory connections on excitatory neurons, scaled by cholinergic modulation $c_{f b}$. The passive decay of membrane potential is scaled to the decay parameter $\eta$ (inverse of membrane time constant). $B$ : for computational modeling of associative memory function, we model a network of interacting subpopulations of pyramidal cells and interneurons. Afferent input patterns $(A)$ consist of binary patterns of excitatory input to pyramidal cell populations in the network. The network contained extensive excitatory feedback connections between interneurons $\left(W_{i j}\right)$ and connections to and from inhibitory interneurons $\left(W^{\prime}\right.$ and $H$ ). In addition, the network contains cholinergic modulation $\Psi$ regulated by inhibitory units influenced by total excitatory output in the network.

excitatory neurons into separate populations representing components of different odor patterns while simulating just one population of inhibitory neurons.

In this computational model of cortical memory function, individual odors are represented as different patterns of afferent input activating specific subpopulations of excitatory neurons (Hasselmo 1995; Hasselmo and Linster 1998a; Hasselmo et al. 1992, 1995; Linster and Hasselmo, 1997). The afferent patterns representing individual odors are stored as self-sustained equilibrium states (attractor states) in the network, with a particular pattern of active neurons within the network. Once an odor is stored as an attractor state, input that resembles that odor attractor state will put the network into the same attractor state. Thus individual differences in neuronal activity (caused by changes in odor concentration or background odors) can be ignored in favor of deciding on a specific odor. Within this general framework, ACh can be seen as altering the sensitivity to external features of the stimulus relative to the internal stored representation. As ACh levels are increased in the network, the relative influence of afferent input increases, allowing greater sensitivity to variations in the external input.

The network used the activation dynamics described by Eq. 2 (as difference equations). These activation dynamics differ from Eq. 1 to include reversal potentials for excitatory and inhibitory synaptic inputs

$$
\begin{aligned}
& \Delta a_{i}=A_{i}-\eta a_{i}+\left(E_{\mathrm{AMPA}}-a_{i}\right) \sum_{j} W_{i j}\left[a_{j}-\theta_{a}\right]_{+} \\
& -\left(E_{\mathrm{GABA}_{\mathrm{A}}}-a_{i}\right) \sum_{l} H_{i l}\left[h_{l}-\theta_{h}\right]_{+} \\
& \Delta h_{k}=A_{k}^{\prime}-\eta h_{k}+\left(E_{\mathrm{AMPA}}-h_{k}\right) \sum_{j} W_{k j}^{\prime}\left[a_{j}-\theta_{a}\right]_{+} \\
& -\left(E_{\mathrm{GABA}_{\mathrm{A}}}-h_{k}\right) \sum_{l} H_{k l}^{\prime}\left[h_{l}-\theta_{h}\right]_{+}
\end{aligned}
$$

Reversal potentials for membrane currents were expressed relative to the resting potential. Thus $E_{\mathrm{AMPA}}=70 \mathrm{mV}$ and $E_{\mathrm{GABAA}}=0 \mathrm{mV}$. Threshold potentials were equivalent for all neurons: $\theta_{\mathrm{a}}=\theta_{h}=8.0$ $\mathrm{mV}$. The size of each time step was one-tenth of a millisecond. The standard time constant of piriform cortex pyramidal cells is $\sim 10 \mathrm{~ms}$ (Barkai and Hasselmo 1994). The decay constant was set at $\eta=0.01$ so that neurons would have a time constant of $10 \mathrm{~ms}$ (units of $\eta$ are in the inverse of 1/10ths of a millisecond). Afferent input was scaled to the magnitude of the decay constant allowing afferent input to depolarize the neurons to $10.0 \mathrm{mV}(A=0.1)$. This can be seen from the equation showing only the effect of afferent input and passive decay $\Delta a=A-\eta a$. In the equilibrium state $\Delta a=0$ and $a=A / \eta$. As noted previously, $A$ represents the product of the output from olfactory bulb mitral cells (in spikes/ms) coming through afferent synapses (with strength in $\mathrm{mV} / \mathrm{spike}$ ). Thus $A$ has units spikes/ms $\times$ $\mathrm{mV} / \mathrm{spike}=\mathrm{mV} / \mathrm{ms} ; a=A / \eta=[\mathrm{mV} / \mathrm{msec}] \times \mathrm{ms}=\mathrm{mV}$. For these simulations, synaptic connectivity took the values $W^{\prime}=0.0008$, and $H=0.0035, H^{\prime}=-0.0055$. Excitatory feedback started at very low values $(W=0.000002)$ and increased to a maximum strength of $W=$ 0.00055 .

Additional equations were utilized to simulate the feedback regulation of cholinergic modulation and the modification of excitatory recurrent synapses for storage of patterns (Hasselmo et al. 1995). Experimental evidence demonstrated that stimulation of cholinergic input from the horizontal limb of the diagonal band influences synaptic transmission in piriform cortex, and stimulation of piriform cortex causes phases of excitation and inhibition in the horizontal limb (Linster et al. 1997). Here we focus on feedback inhibition of cholinergic modulation. ACh levels were represented by $\psi$ and depended on a threshold linear function $\Psi[\alpha-\theta]_{+}$of the average membrane potential $\alpha$ of a population of cholinergic neurons 


$$
\begin{gathered}
\psi=\Psi\left[\alpha-\theta_{\alpha}\right]_{+} \\
\Delta \alpha=\mathrm{A}_{\psi}-\eta \alpha-H_{\psi}\left[h-\theta_{h}\right]_{+}
\end{gathered}
$$

where $\theta_{\alpha}$ is the output threshold for the population of cholinergic neurons in the horizontal limb, $A_{\psi}$ is tonic input to the cholinergic neuron present at all times during simulations to ensure continuous output in the absence of inhibition, and $H_{\psi}$ is inhibitory input to cholinergic neurons from GABAergic neurons activated by piriform cortex pyramidal cells. Simulations used the values $A_{\psi}=0.3, \theta_{\alpha}=$ 8.0, and $\theta h=8$.

Excitatory feedback connections were modified according to learning rules dependent on postsynaptic activity $a_{i}$ and presynaptic activity $a_{j}$, in keeping with experimental evidence on associative long-term potentiation in piriform cortex (Patil et al. 1998). Modification depended on cumulative buildup of pre- and postsynaptic variables $s_{i}$ and $s_{j}$, which increased with separate dynamics (dependent on an accumulation constant $\phi$ and a diffusion constant $\beta$. This could be construed as the buildup of pre- and postsynaptic calcium or activation of pre- and postsynaptic second messengers such as protein kinase $\mathrm{C}$. The learning rule also contained synaptic decay proportional to the current strength $W_{i j}$ and pre- or postsynaptic activity (scaled with the constants $\omega_{\text {pre }}$ and $\omega_{\text {post }}$ ) as a representation of long-term depression (Levy et al. 1990). Each rule had parameters for the overall modification rate $\kappa$ and the postsynaptic modification threshold $\theta_{w}$. The rate of synaptic modification was also scaled to the level of cholinergic modulation, as suggested by experiments showing cholinergic enhancement of long-term potentiation in piriform cortex (Hasselmo and Barkai 1995; Patil et al. 1998) and hippocampus (Burgard and Sarvey 1990; Huerta and Lisman 1993). The cumulative learning rule took the form

$$
\begin{gathered}
\Delta W_{i j}=\kappa\left(1-\chi_{w} \psi\right)\left(\left[s_{i}-\theta_{w}\right]_{+}-\omega_{\mathrm{pre}} W_{i j}\right)\left(\left[s_{j}-\theta_{w}\right]_{+}-\omega_{\mathrm{post}} W_{i j}\right) \\
\Delta s_{i}=\phi\left[a_{i}-\theta_{a}\right]_{+}-\beta s_{i} \\
\Delta s_{j}=\phi\left[a_{j}-\theta_{a}\right]_{+}-\beta s_{j}
\end{gathered}
$$

Connections to and from inhibitory interneurons were not modified in these simulations. In most simulations, weights were clipped at specific values to maintain them within the region of stable attractor dynamics. This clipping allowed stable learning for a broader range of parameters.

In this computational model, a set of binary input patterns represented the neuronal activity associated with a series of different odors. These binary input patterns were stored in the network through Hebbian modification of excitatory association connections. The storage of these patterns was subsequently evaluated by presenting one portion of the input patterns and determining if the network could complete the missing components of the input patterns. A performance measure based on normalized dot products was used to evaluate the effectiveness of retrieval, as in previous articles (Barkai et al. 1994; Hasselmo et al. 1992). This performance measure increased with the effective completion of a learned pattern but decreased with the similarity between the learned pattern and other learned patterns. In this simulation, the network is first trained on one input pattern (1010010010) followed by another input pattern with considerable overlap (0101010010), as shown in Fig. 4. In the simulations shown here, high performance occurs when the first pattern is learned properly but does not interfere with learning of the second pattern. The performance measure is very low when no learning occurs and retrieval activity is low. The performance measure also goes to low values when the first pattern interferes with learning of the second, causing the response to the degraded input to contain elements of both the stored patterns.

\section{Brain slice physiology}

Intracellular recordings (with sharp electrodes in current-clamp mode and patch electrodes in current- and voltage-clamp modes) were obtained from pyramidal neurons in layer II of the piriform cortex in the in vitro slice preparation. Slices $400 \mu \mathrm{m}$ thick were obtained from adult female Sprague-Dawley rats $(150-200 \mathrm{gm})$, by using standard procedures (Hasselmo and Barka, 1995; Hasselmo and Bower 1992) in accordance with institutional guidelines. The animal was lightly anesthetized with halothane and decapitated. The brain was rapidly removed and placed in chilled oxygenated artificial cerebrospinal fluid (ACSF) maintained close to $4^{\circ} \mathrm{C}$. Slices were cut in the coronal plane, perpendicular to the laminar organization of the piriform cortex, with a vibratome. Once cut, the slices were stored at room temperature in a chamber containing oxygenated ACSF solution with the following composition (in mM): $124 \mathrm{NaCl}, 5 \mathrm{KCl}, 1.2 \mathrm{KH}_{2} \mathrm{PO}_{4}, 1.3 \mathrm{MgSO}_{4}, 2.4$ $\mathrm{CaCl}_{2}, 26 \mathrm{NaHCO}_{3}$, and 10 D-glucose (pH 7.4-7.5).

After $1 \mathrm{~h}$ of incubation at room temperature slices were placed on a nylon mesh in a submerged chamber with oxygenated ACSF flowing over the slices at a rate of $1 \mathrm{ml} / \mathrm{min}$. A thermistor, placed just below the mesh, monitored and controlled the temperature of the ACSF through a temperature regulator circuit maintaining it between 33 and $34^{\circ} \mathrm{C}$. Intracellular responses to electrical stimuli were recorded once the pyramidal neurons were impaled. All recorded neurons had a resting membrane potential of $-65 \mathrm{mV}$ or more negative.

The orthodromic stimuli were delivered through a NeuroData PG4000 stimulator, with two fine unipolar tungsten electrodes, one positioned in the intrinsic layer and other in the afferent layer. The brain slice preparation is illustrated in Fig. 2. For sharp electrode recording, the glass micropipette electrodes (resistance $=70-180$ $\mathrm{M} \Omega$ ) were filled with $4 \mathrm{M}$ potassium acetate solution. In four neurons QX314 (50 mM) was introduced to block the sodium spikes and the slow-inhibitory postsynaptic potential (IPSP) component to better record the fast-IPSP component. From these four neurons only IPSP data were recorded; they were not used for threshold firing values. IPSPs were primarily recorded by intracellular current injection during association-afferent fiber stimulation, as shown by Tseng and Haberly (1988). The fast and slow IPSP components, shown to be mediated by changes in $\mathrm{Cl}^{-}$and $\mathrm{K}^{+}$conductances, respectively (Tseng and Haberly 1988), were measured at time periods corresponding to the maximum IPSP peaks in control (between 20 and $40 \mathrm{~ms}$ for fast and between 100 and $140 \mathrm{~ms}$ for slow component). The same time reference was used in individual neurons, but it varied slightly over the population. Orthodromic thresholds were examined by varying the strength of the stimulus at certain stimulus durations $(0.2,0.5,1.5$, and $3 \mathrm{~ms}$ in most cells). Input resistance was calculated from the responses to 200-ms hyperpolarizing current pulses.

Whole cell patch recordings for both current clamp and voltage clamp were carried out with borosilicate glass electrodes, pulled to give an electrode resistance of $\sim 8-12 \mathrm{M} \Omega$. The electrode solution had the following composition (in $\mathrm{mM}$ ): $120 \mathrm{~K}$-gluconate, $10 \mathrm{KCl}, 2$ $\mathrm{CaCl}_{2}, 2 \mathrm{MgCl}_{2}, 2$ EGTA, $2 \mathrm{~K}$-ATP, 0.2 Na-GTP, 20 HEPES,

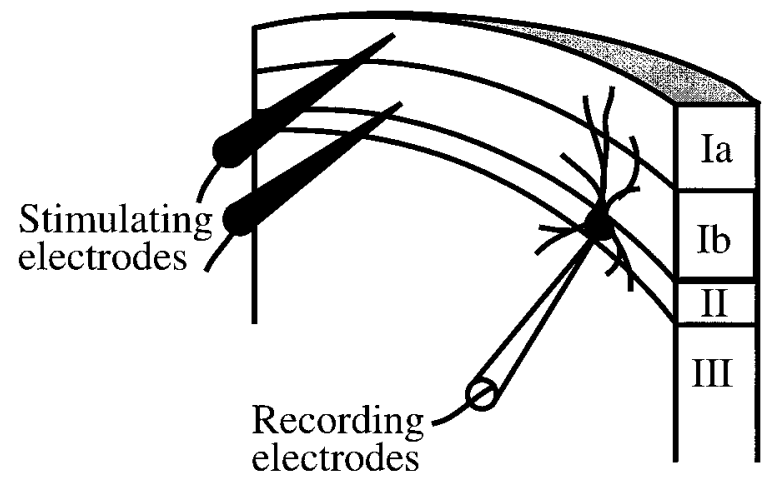

FIG. 2. Brain slice preparation of the piriform cortex, showing location of stimulating electrodes in layer Ia and layer Ib. Intracellular recordings were obtained from pyramidal cell bodies in layer II. This allowed analysis of excitatory and inhibitory synaptic potentials elicited by stimulation through electrodes located in either layer Ia or layer Ib. 
buffered to pH-7.2 with $\mathrm{KOH}$, having an osmolarity of 280 mosm. The solution was filtered before filling the electrodes. An Axoclamp-2A amplifier was used for recordings, and data acquisition was performed with pClamp 6.4 software and Digidata 1200 interface board. Data were sampled at 10 or $20 \mathrm{kHz}$, depending on the nature of the responses. Unless otherwise noted, holding potential for voltage-clamp recording of synaptic currents was $-60 \mathrm{mV}$.

Effects of cholinergic modulation were studied by the introduction of the cholinergic agonist carbachol (carbamylcholine chloride, $\mathrm{CCh}$ ) into the bathing medium at a concentration of $50 \mu \mathrm{M}$. To isolate IPSPs, excitatory postsynaptic potentials (EPSPs) were blocked with the introduction of $\mathrm{D}(-)$-2-amino-5-phosphonovaleric acid (D-APV) at $40 \mu \mathrm{M}$ and 6-cyano-7-nitroauinoxaline-2,3-dione (CNQX) at 20 $\mu \mathrm{M}$ into the bath for some cells before the application of CCh. The drugs were obtained from Research Biochemicals (Natick, MA). Data were analyzed with two-way ANOVA. Results are quantified as the means $\pm \mathrm{SE}$, and the accepted level for significance was $P<0.05$ (unless otherwise stated).

\section{R E S U L T S}

\section{Mathematical analysis}

As described in METHODS, the simplified network allowed mathematical analysis of how much modulation of feedforward and feedback inhibitory synaptic transmission would be necessary to offset the previously observed modulation of excitatory synaptic transmission (Hasselmo and Bower 1992). This analysis was performed with the network with a single unit representing average membrane potential $a$ of a subpopulation of excitatory neurons and a single unit representing average membrane potential $h$ of the population of inhibitory neurons (see Fig. 1A).

By using the equations shown in METHODS, we can solve for the average membrane potential within a subpopulation of excitatory neurons when the network is in equilibrium. This could correspond to a particular self-sustained memory state toward which the network evolves (an attractor state), or it could simply represent the steady-state response to a particular new odor. In either case, the equilibrium can be determined by observing the state of the equations when there is no change in the value of $a$ or $h$, i.e., by setting da/dt $=\mathrm{dh} / \mathrm{dt}=0$ (Hasselmo et al. 1995). Real biological networks probably only enter equilibrium states for brief periods, but the network may be continuously moving toward particular stable equilibrium states. Equilibrium states can determine the level of network activity even when there are slow oscillatory changes in particular parameters (Tsodyks et al. 1997). The value of $a$ during this equilibrium state is obtained by algebraically solving for $a$ after setting $\mathrm{da} / \mathrm{dt}=\mathrm{dh} / \mathrm{dt}=0$ in the equations presented in METHODS. The average excitatory membrane potential during this equilibrium state will be designated as $Q$.

$$
Q=a_{e q}=\frac{A-W \theta_{a}+\frac{H W^{\prime} \theta_{a}}{\eta^{\prime}}+H \theta_{h}}{\eta-W+\frac{H W^{\prime}}{\eta^{\prime}}}
$$

As noted in METHODS, $A=$ afferent input, $W=$ excitatory connections between pyramidal cells, $\theta_{a}=\theta_{h}=$ threshold of pyramidal cells and interneurons, $H=$ inhibitory connections to pyramidal cells, $W^{\prime}=$ excitatory connections to inhibitory interneurons, and $\eta=\eta^{\prime}=$ inverse time constant of pyramidal cells and interneurons. This equation can be simplified if we assume that afferent input $A$ is always strong enough alone to bring the average membrane potential above threshold. If there were no synaptic connections (if $W=W^{\prime}=H=0$ ), then the equilibrium state would be $Q=A / \eta$. Because afferent input initiates activity in the network, we make the assumption that afferent input alone can bring neurons over threshold, so $A / \eta>$ $\theta$. To assist in simplifying the equation, we can split the strength of $A$ into a quantity that brings the neurons up to threshold (rearranging the equation $A / \eta=\theta$, we obtain $A=$ $\eta \theta)$ and an additional quantity $(X)$ that goes beyond threshold. Then we write $A$ as $X+\eta \theta$. In addition, to include feedforward inhibition, we will represent the suprathreshold afferent input $X$ as a combination of direct input $I$ and a feedforward inhibition scaled to the direct input (this assumes that feedforward inhibitory input cannot cause afferent input to become subthreshold). Thus $A=I-H_{f f} I+\eta \theta$. This gives us the following equilibrium state $Q$

$$
Q=\frac{I-H_{f f} I+\eta \theta-W \theta_{a}+\frac{H W^{\prime} \theta_{a}}{\eta^{\prime}}+H \theta_{h}}{\eta-W+\frac{H W^{\prime}}{\eta^{\prime}}}=\frac{I-H_{f f} I+H \theta_{h}}{\eta-W+\frac{H W^{\prime}}{\eta^{\prime}}}+\theta
$$

By using this equation, we analyzed how cholinergic modulation of the strength of recurrent excitation $W$ (represented by the suppression variable $c_{w}$ ) could be offset by cholinergic modulation of other parameters, allowing the network to maintain the same value of $a$ in the equilibrium state. As noted previously, the network may change somewhat in level of activity during cholinergic modulation but does not become inactive or go into unstable seizure activity. As will be seen in the following sections, there is a striking qualitative difference in how well modulation of different inhibitory connections $(H$ or $H_{f f}$ ) can compensate for the activity changes associated with suppression of excitatory intrinsic connections $(W)$. Thus this analysis is relevant even if the goal is only to keep membrane potentials and network activity within a general range of values.

In each of the following sections, the relationship between $c_{w}$ and one of the other modulation variables is analyzed (setting other variables equal to one). These sections address 1) the suppression of feedforward inhibition $H_{f f}$ (suppressed in proportion to the variable $c_{f f}$ ), 2) the suppression of feedback inhibition $H$ (suppressed in proportion to the variable $c_{f b}$ ), and 3 ) the suppression of the excitatory input to feedback interneurons $W^{\prime}$ (suppressed in proportion to the variable $c_{w^{\prime}}$ ). In each of these sections, we set the equilibrium state with cholinergic modulation equal to the equilibrium state without cholinergic modulation, as follows

$$
Q=\frac{I-c_{f f} H_{f f} I+c_{f b} H \theta_{h}}{\eta-c_{w} W+\frac{c_{f b} H c_{w^{\prime}} W^{\prime}}{\eta^{\prime}}}+\theta=\frac{I-H_{f f} I+H \theta_{h}}{\eta-W+\frac{H W^{\prime}}{\eta^{\prime}}}+\theta
$$

\section{Relationship between recurrent excitation $(W)$ and} feedforward inhibition $\left(H_{f f}\right)$

This section explores how much modulation of feedforward inhibition $c_{f f}$ can compensate for changes in the modulation of feedback excitation $c_{w}$ to maintain approximately the same level of average membrane potential during the equilibrium state of the network. On an intuitive level, the loss of excitation 
in the network should cause a decrease in activity, but this decrease in activity could be prevented if there is a corresponding decrease in inhibition. For example, if a subpopulation of excitatory neurons has a certain activity level in response to a particular odor and we then alter the strength of feedback excitation caused by cholinergic modulation, can a change in feedforward inhibition keep the network activity at approximately the same average level? For the moment, the cholinergic modulation of feedback inhibition and input to interneurons will be ignored, so we will keep $c_{f b}=c_{W^{\prime}}=1$. We can then rearrange $E q .7$ to see how much $c_{f f}$ must change during changes in $c_{w}$ to prevent cholinergic modulation from causing a change in the average level of activity $(Q)$. Algebraic manipulation of $E q .7$ yields the following relation

$$
c_{f f}=\frac{\left(c_{w}-1\right)(I W+H W \theta)+H_{f f} I\left(\eta-c_{w} W+H_{f f} H W^{\prime} / \eta\right)}{H_{f f} I\left(\eta-c_{w} W+H W^{\prime} / \eta\right)}
$$

Both $c_{f f}$ and $c_{w}$ must remain between 0 and 1 because 0 represents complete suppression of synaptic transmission, and 1 represents normal levels of transmission. In fact, physiologically realistic maximal suppression is probably more in the range of $c_{w}=0.3$ (Hasselmo and Bower 1992). However, in $E q .8, c_{f f}$ rapidly must go to 0 to compensate for even very small decreases in $c_{w}$. We can explore the maximal effect of cholinergic suppression of feedforward inhibition if we set $c_{f f}$ to 0 (meaning feedforward inhibition is completely suppressed by cholinergic modulation) and assume that $H_{f f}$ approaches 1.0 (meaning that it is very strong in the absence of cholinergic modulation). In this case, when $c_{f f}$ is 0 , it compensates for $c_{w}$ at the following value

$$
c_{w}=1-\frac{I\left(\eta-W+H W^{\prime} / \eta\right)}{H W \theta}
$$

Putting specific numerical parameters into this equation shows that the range of $c_{w}$ for which $c_{f f}$ can compensate is very small. As an example, we can use parameters from previous research (Hasselmo et al. 1995) in which stable equilibrium states were described with $W=0.016, H=0.06, W^{\prime}=0.0042$, and $\eta=\eta^{\prime}=0.01$ (that paper used $A=0.1$, corresponding to $I=0.02$ ). The use of these specific numerical parameters is significant because only a certain number of parameters yield stable equilibrium states that could correspond to memory states (Hasselmo et al. 1995). For these parameters, as we change the value of $c_{w}$ from 1.0 to 0.95 , we can keep the average equilibrium membrane potential in the same range by changing $c_{f f}$ from 1.0 to 0.0 . However, this only compensates for a small change in feedback excitation. As feedback excitation $W$ is reduced by values of $c_{w}$ smaller than 0.95 , changes in feedforward inhibition cannot further compensate.

If $I$ is increased greatly, $c_{f f}$ can compensate for a wider range of values of $c_{w}$, but great increases in $I$ are inconsistent with the distal termination of afferent synapses on piriform cortex pyramidal cells. In summary, modulation $c_{f f}$ of feedforward inhibition $H_{f f}$ is not an effective means to compensate for modulation $c_{w}$ of recurrent excitation $W$. This generates the prediction that there probably is not a strong effect of cholinergic suppression of inhibitory potentials in layer Ia of the piriform cortex.
Relationship between recurrent excitation $(W)$ and feedback inhibition $(H)$

We can also compensate for changes in the recurrent excitation $W$ with changes in the inhibitory synaptic transmission $H$ arising from interneurons activated by feedback from pyramidal cells. In this case, we change the levels of feedback inhibitory transmission $H$ according to the modulation parameter $c_{f b}$ while keeping other modulatory parameters at $c_{f f}=$ $c_{W^{\prime}}=1$. By algebraically rearranging $E q$. 7, we obtain the following relation

$$
c_{f b}=\frac{\left(1-c_{w}\right) I\left(1-H_{f f}\right) W+H \theta\left(\eta-c_{w} W\right)}{H \theta(\eta-W)}
$$

As in the previous discussion, both $c_{f b}$ and $c_{w}$ must remain between 0 and 1 . We can explore the maximal effect of cholinergic suppression of feedback inhibitory transmission if we set $c_{f b}$ to 0 . When $c_{f b}$ is 0 , it compensates for $c_{w}$ at the following value

$$
c_{w}=\frac{H \theta \eta+I\left(1-H_{f f}\right) W}{H \theta W+I\left(1-H_{f f}\right) W}
$$

The range of $c_{w}$ for which $c_{f b}$ can compensate is larger than that for which $c_{f f}$ can compensate. As an example, we can use the same parameters from previous research (Hasselmo et al. $1995)$ as in the previous section. For these parameters, $c_{f b}$ can compensate for values of $c_{w}$ between 1.0 and 0.64. (i.e., $c_{f b}$ must be set at 0 to compensate for $c_{w}=0.64$ ) If $I$ is increased greatly, this range decreases, consistent with the change in relative influence of afferent versus intrinsic synapses. This suggests that suppression of feedback inhibition in the cortex can more effectively compensate for changes in feedback excitation than changes in feedforward inhibition. In the piriform cortex, where feedback excitation has been shown to be suppressed, this suggests that there should be an accompanying suppression of feedback inhibition.

\section{Relationship between recurrent excitation $(W)$ and excitatory input to feedback interneurons $\left(W^{\prime}\right)$}

We can also compensate for changes in the recurrent excitation $W$ with changes in the excitatory input $W^{\prime}$ to interneurons mediating feedback inhibition. In this case, we change the level of excitatory input to interneurons according to the modulation parameter $c_{w^{\prime}}$, setting $c_{f f}=c_{f b}=1$. From $E q$. 7, we obtain the following relation

$$
c_{w^{\prime}}=1+\left(c_{w}-1\right) \frac{W \eta}{H W^{\prime}}
$$

We can explore the maximal effect of cholinergic suppression of input to feedback interneurons if we set $c_{w^{\prime}}$ to 0 . When $c_{w^{\prime}}$ is 0 , it compensates for $c_{w}$ at the following value

$$
c_{w}=1-\frac{H W^{\prime}}{\eta W}
$$

Changes in $c_{w^{\prime}}$ can compensate for a very wide range of values of $c_{w}$. In fact, when we use the parameters from previous research (Hasselmo et al. 1995), we see that changing the value of $c_{w^{\prime}}$ from 1.0 to 0.365 can compensate for changing the value of $c_{w}$ from 1.0 to 0.0 . At $c_{w^{\prime}}=0.365$ the suppression of excitatory input to interneurons can compensate for $c_{w}=$ 
0.0. Thus smaller changes in modulation of excitatory input to interneurons can compensate for larger changes in modulation of excitatory input to other pyramidal cells.

When considering this analysis of equilibrium state, we should consider not only the value of the equilibrium state but also the stability of this equilibrium state. Mathematical analysis demonstrates which parameters allow stable self-sustained equilibrium states. The value of $c_{w}=0$ is not realistic for equilibrium states because the equilibrium state becomes unstable when the excitatory connection between pyramidal cells $\left(c_{w} \times W\right)$ drops below the rate of passive membrane potential decay $\eta$. On an intuitive level, a network will never be able to sustain activity if the neurons lose membrane potential more rapidly than feedback excitation can build it up. To maintain equilibrium with these parameters, the feedback excitation $\left(c_{w} \times W\right)$ needs to be larger than the membrane potential decay $\eta=0.01$. This occurs at $c_{w}=0.625$, which can be compensated by $c_{w^{\prime}}=0.762$. Only the suppression of excitatory input to interneurons can compensate for the full range of $c_{w}$ values for which the equilibrium remains stable. Thus suppression of excitatory input to interneurons can be weaker than suppression of excitatory transmission between pyramidal cells. In contrast to both the other examples presented previously, this compensation also does not change for different values of the afferent input $I$, suggesting that it might be easier to implement. This suggests that compensation of changes in feedback excitation with changes in feedback inhibition may depend strongly on changes in the excitatory input to inhibitory interneurons rather than just on changes in the inhibitory transmission from interneurons. Inhibitory synaptic potentials evoked in layer $\mathrm{Ib}$ of the piriform cortex in the experiments described here contain components of both $W^{\prime}$ and $H$. Thus the analysis suggests that we should see a much stronger cholinergic suppression of inhibitory synaptic potentials in layer Ib than in layer Ia.

\section{Relationship between depolarization of interneurons $\left(A^{\prime}\right)$ and excitatory input to interneurons $\left(W^{\prime}\right)$}

Cholinergic modulation enhances the frequency of spontaneous inhibitory synaptic currents during recordings from pyramidal cells in the hippocampus (Behrends and ten Bruggencate 1993; Pitler and Alger 1992). This frequency increase is believed to result from direct cholinergic depolarization of GABAergic interneurons (McQuiston and Madison 1996), which would increase firing rate. This depolarization of interneurons appears rather paradoxical when combined with the suppression of evoked inhibitory potentials. Why would the same substance simultaneously increase inhibition via direct depolarization while suppressing total feedback inhibition? The analytic framework presented here provides a possible explanation of this paradox. Starting with Eq. 1, we can analyze the effect of depolarization of inhibitory interneurons by representing it as a direct depolarizing afferent input to interneurons $A^{\prime}$, yielding the following equilibrium state

$$
Q=\frac{I-A^{\prime} H / \eta^{\prime}-H_{f f} I+H \theta_{h}}{\eta-W+\frac{H W^{\prime}}{\eta^{\prime}}}+\theta
$$

As shown in Fig. $3 A$, increases in this direct depolarization of interneurons $A^{\prime}$ will shift the equilibrium state of the network downward across the full range of values for input to the
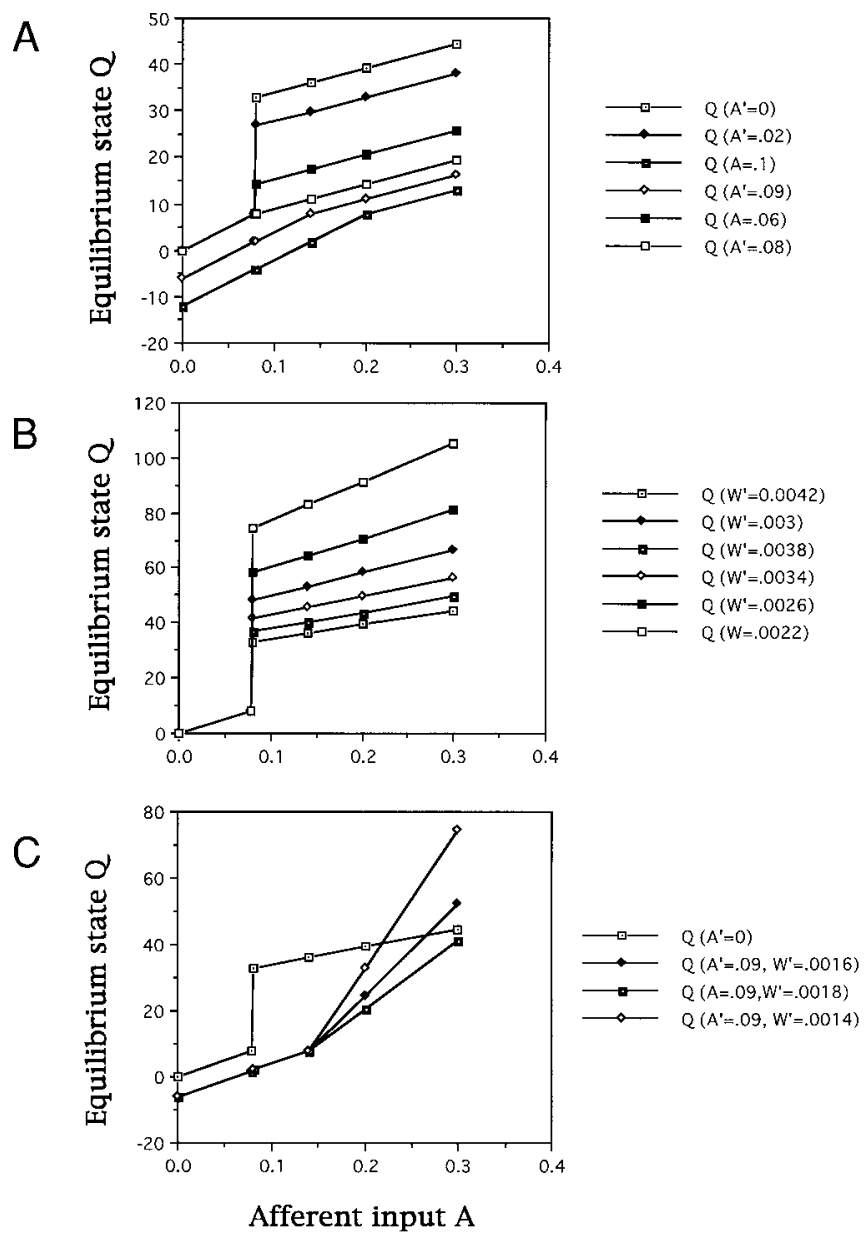

FIG. 3. Effect of modulation on equilibrium state in the network shown in Fig. $1 A$. Equilibrium state $Q$ is shown for different values of excitatory afferent input $A$ to the unit representing the excitatory subpopulation. Low values of $A$ do not put the average membrane potential a over threshold. This subthreshold equilibrium state increases linearly, depending only on the decay time constant of excitatory neurons. When the membrane potential of the excitatory population reaches threshold, the equilibrium state increases dramatically because of excitatory feedback in the network, but a further increase in afferent input $A$ results in further linear increases in equilibrium state $Q$. $A$ : increases in the depolarizing input to interneurons $A^{\prime}$ shifts the equilibrium response downward. For subthreshold levels of interneuron input $\left(A^{\prime}<0.8\right)$ only the equilibrium states for suprathreshold values of $A$ are affected, but for suprathreshold levels of interneuron input $\left(A^{\prime}>0.8\right)$ the subthreshold response of excitatory neurons is affected, preventing them from becoming active at low values of $A$. B: decreases in $W^{\prime}$ change the magnitude and increase the slope of the suprathreshold response. $C$ : combination of increases in $A^{\prime}$ (depolarization of interneurons) with decreases in $W^{\prime}$ (suppression of excitatory input to interneurons) makes the network respond more weakly to weak input (low values of $A$ ) but more strongly to strong input (high values of $A$ ).

excitatory neurons $A$. For values of $A^{\prime}$ that cause spontaneous spiking of the interneurons this requires an increased amount of afferent input $A$ to get some response in the excitatory population. Thus, although there is no change in the threshold value $\theta$, the functional threshold is increased. As shown in Fig. 3B, suppression of $W^{\prime}$ will alter the slope of the relationship between afferent input $A$ and the equilibrium state $Q$, resulting in a steeper slope. As shown in Fig. $3 C$, combination of direct depolarization with suppression of $W^{\prime}$ can result in a network that responds less to weak inputs but responds more strongly to stronger afferent input. Thus these two effects could interact to 
make the spontaneous and background activity of the network weaker, while enhancing the response to strong afferent input.

\section{Computational modeling}

As described in METHODS, the effect of cholinergic modulation of inhibitory synaptic potentials was also tested in a computational model of associative memory function. In this model, the storage of highly overlapping input patterns was analyzed with different levels of cholinergic modulation of synaptic transmission. This gives a notion of how cholinergic modulation affects the overall function of the network. The model shows that cholinergic suppression of feedback inhibition is necessary for effective function, whereas the cholinergic suppression of feedforward inhibition does not have a strong role in ensuring effective function. In fact, previous simulations of associative memory function used selective cholinergic suppression of feedback but not feedforward inhibition (Hasselmo et al. 1995), although the full range of parameter values was not previously explored.

Figure 4 demonstrates the basic function of the network for different values of cholinergic modulation of feedback inhibition $H$. Each section of the figure shows the activity in a network of 10 excitatory neurons and 1 inhibitory neuron during sequential presentation of different patterns of input (1010010010 and 0101010010). Pattern number one is first presented, followed by a degraded version of that input pattern. Then a second pattern that overlaps with the first pattern is presented, followed by a degraded version of that second pattern. For each pattern, the activity of the network is shown during a number of time steps. The width of black lines represents the activity of individual neurons within the network. For insufficient cholinergic suppression of inhibitory feedback $\left(c_{f b}=0.6\right)$, inhibition is too strong in the network, and the degraded patterns evoke activity in only two of the normal four neurons. For excessive cholinergic suppression of inhibitory feedback $\left(c_{f b}=1.0\right)$, inhibition is insufficient, and there is severe interference between highly overlapping stored patterns. This results from the fact that some inhibition is necessary during learning to prevent interference in the network (Hasselmo 1993). Appropriate levels of the cholinergic suppression of inhibitory feedback $\left(c_{f b}=0.8\right)$ provides effective function in the model. In this case, the network responds to the degraded version of the input patterns with the full learned version of those input patterns.

\section{Suppression of feedback inhibitory transmission $(H)$ and feedforward inhibition $\left(H_{f f}\right)$}

As shown in Fig. 5A suppression of feedback inhibitory transmission $(H)$ is more important for good memory performance in the model than suppression of feedforward inhibition $\left(H_{f f}\right)$. Simulations were used to evaluate the memory performance of the network for a large number of different values of suppression of inhibitory transmission (the influence of interneurons on pyramidal cells) in combination with a large number of different values for suppression of feedforward inhibition. As can be seen in the figure, the best performance occurred with high levels of suppression of inhibitory transmission $(H)$, ranging between 65 and $100 \%$ suppression (corresponding to $\left.c_{f b}=0.35-0.0\right)$. As can be seen from the graph,
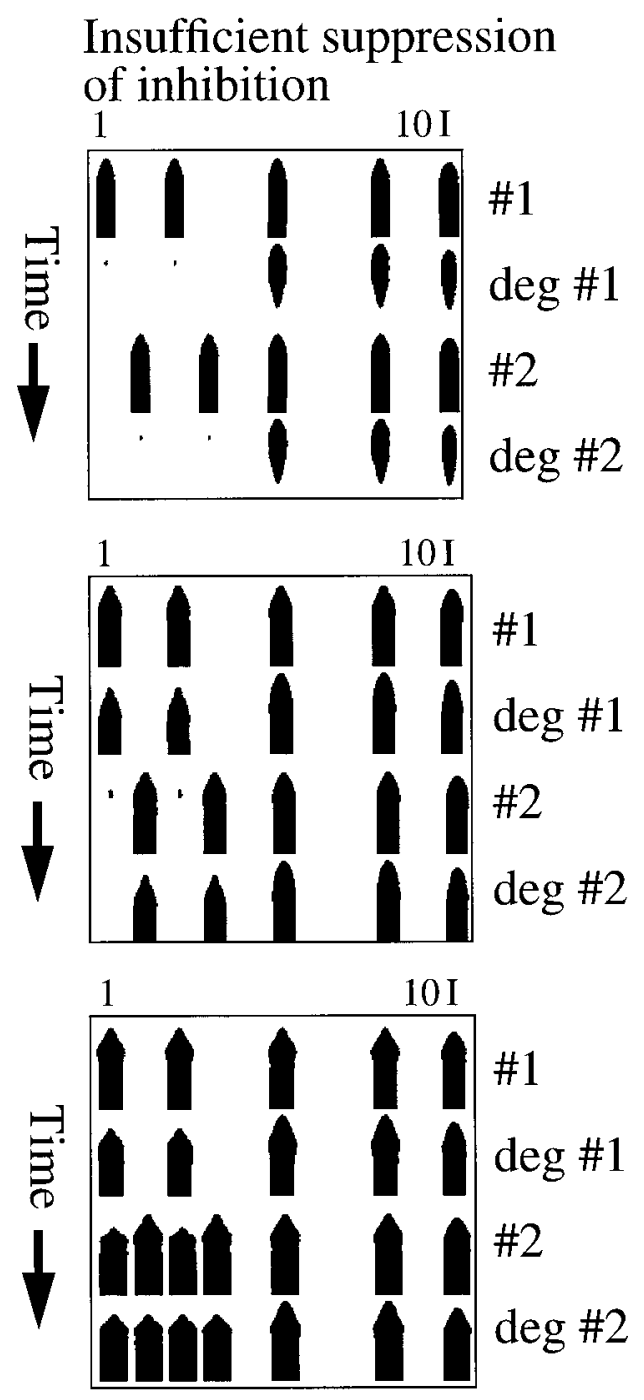

\section{Excessive suppression of inhibition}

FIG. 4. Associative memory function in the network with different levels of cholinergic suppression of feedback inhibition $H$. Each section of the figure shows the activity across time (plotted vertically) in a network of 10 excitatory units (1-10 graphed horizontally, each representing a population of neurons) and 1 inhibitory unit $(I)$ representing the inhibitory population during sequential presentation of different patterns of input. For each pattern, the activity of the network is shown during a number of time steps. The width of black lines represents the activity of individual units within the network. Pattern number 1 is first presented (\#1), followed by a degraded version of that input pattern (deg \#1). A second pattern that overlaps with the first pattern is then presented (\#2), followed by a degraded version of that second pattern (deg \#2). Note that, for insufficient cholinergic suppression of inhibitory feedback, inhibition is too strong in the network, and the degraded patterns evoke activity in only 2 of the normal 4 units. For excessive cholinergic suppression of inhibitory feedback, inhibition is insufficient, and there is severe interference between highly overlapping stored patterns.

suppression of feedforward inhibition does not as strongly influence levels of performance. High levels of performance could be obtained across all values for feedforward inhibition, although lower levels of suppression of feedback inhibitory transmission were necessary when there was stronger suppression of feedforward inhibition. The results from these simulations suggest that for effective memory performance strong 
suppression of feedback inhibition is more necessary than suppression of feedforward inhibition.

\section{Suppression of excitatory input to interneurons $\left(W^{\prime}\right)$ and feedforward inhibition $\left(H_{f f}\right)$}

As shown in Fig. 5B, the effect of suppression of excitatory input to interneurons is similar to the effect of suppression of inhibitory transmission, with a somewhat broader range of good performance. Thus good performance can be obtained with suppression of excitatory input to interneurons $\left(W^{\prime}\right)$ set at $30 \%$ (corresponding to $c_{w^{\prime}}=\leq 0.7$ ). However, this lesser requirement for suppression of excitatory input to interneurons

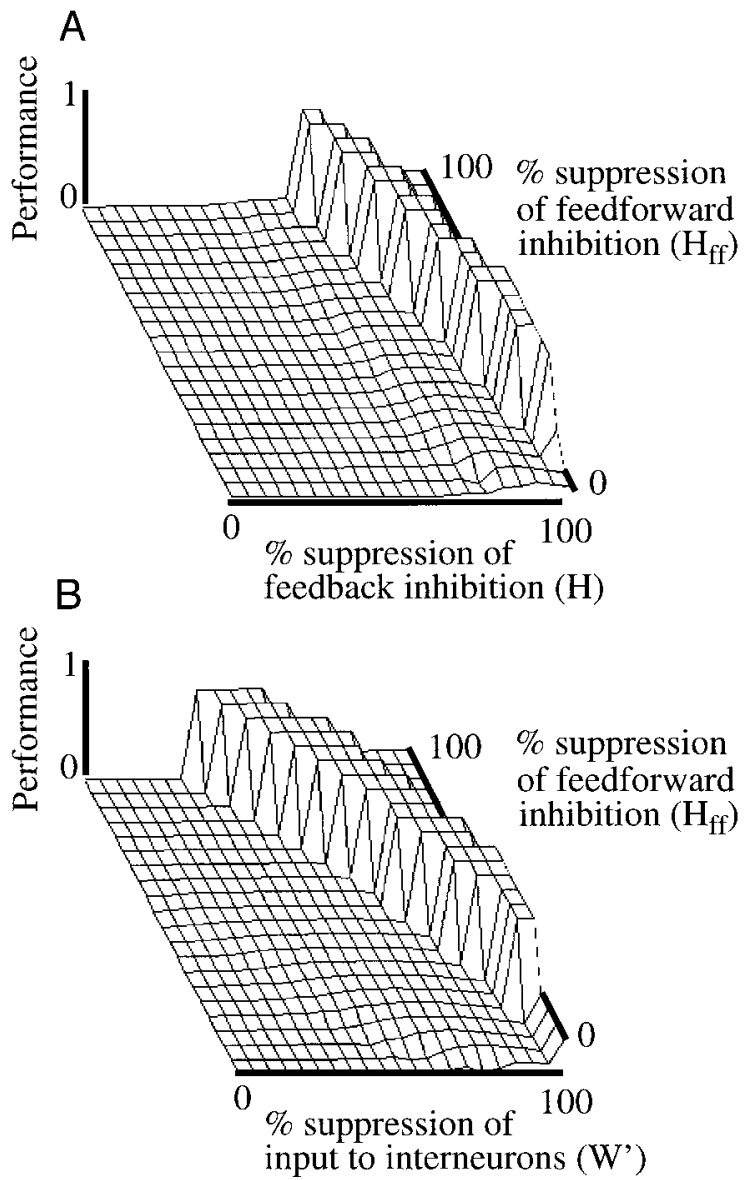

FIG. 5. A: performance of the network across different values of the suppression of feedforward inhibition $\left(H_{f f}\right)$ and the suppression of feedback inhibitory transmission $\left(H_{f b}\right)$. Performance is plotted on the $z$-axis, varying between 0 and 1 . Percentage suppression of inhibitory transmission between inhibitory units and excitatory units is plotted on the $x$-axis, ranging from 0 to $100 \%$. The best performance occurs when suppression of feedback inhibition is $>65 \%$. Percentage suppression of feedforward inhibition is plotted on the $y$-axis, ranging from 0 to $100 \%$. Good performance occurs for the full range of values of suppression of feedforward inhibition, although less suppression of feedback inhibition is necessary when there is greater suppression of feedforward inhibition. $B$ : performance of the network across different values of the suppression of feedforward inhibition $\left(H_{f f}\right)$ and the suppression of excitatory input to interneurons $\left(W^{\prime}\right)$. Percentage suppression of connections from excitatory neurons to interneurons is plotted on the $x$-axis, ranging from 0 to $100 \%$. Note that the best performance occurs when suppression of excitatory input to interneurons is $>30 \%$. Percentage suppression of feedforward inhibition is plotted on the $y$-axis, ranging from 0 to $100 \%$. Good performance occurs for the full range of values of suppression of feedforward inhibition, although less suppression of input to inhibitory units is necessary when there is greater suppression of feedforward inhibition.
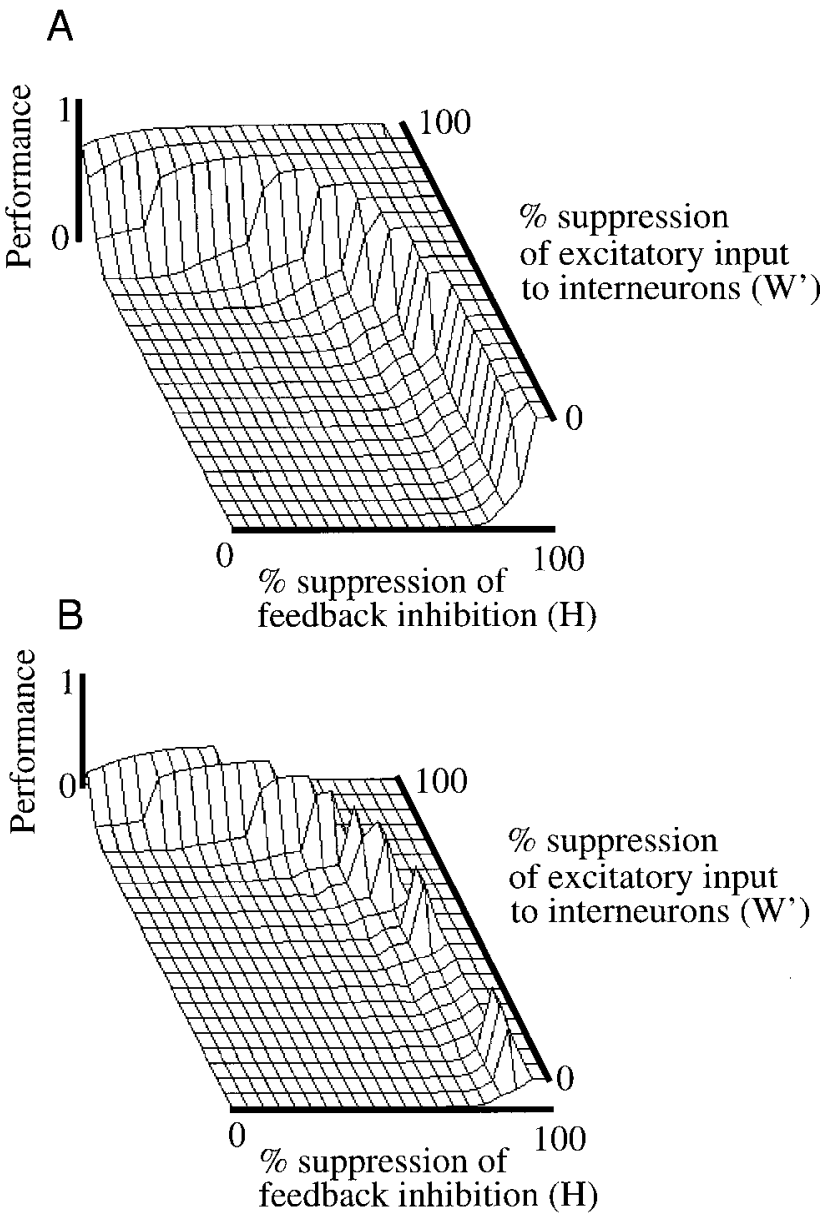

FIG. 6. A: performance of the network across different values of the suppression of feedback inhibitory transmission $\left(H_{f b}\right)$ and the suppression of excitatory input to inhibitory units $\left(W^{\prime}\right)$. Performance is plotted on the $z$-axis, varying between 0 and 1 . Percentage suppression of inhibitory transmission between inhibitory units and excitatory units is plotted on the $x$-axis, ranging from 0 to $100 \%$. Good performance occurs when suppression of either component of feedback inhibition is strong. $B$ : performance of the network for highly overlapping patterns. In this case, when suppression of feedback inhibition becomes too strong, then interference between stored patterns causes a greater decrease in performance, resulting in only a narrow range of effective performance.

only occurs when the suppression of feedforward inhibition is quite strong. Thus for this parameter there is a greater interaction between the level of feedforward inhibition and the level of feedback inhibition, although good function can still be obtained at all values of feedforward inhibition.

\section{Suppression of excitatory input to interneurons $\left(W^{\prime}\right)$ and inhibitory transmission $(H)$}

Figure $6 \mathrm{~A}$ shows the performance of the network for different values of the cholinergic suppression of both components of feedback inhibition, the suppression of excitatory input to interneurons $\left(W^{\prime}\right)$ and the suppression of inhibitory transmission from interneurons to excitatory neurons $(H)$. As can be seen in the figure, effective performance depends on strong modulation of feedback inhibition, but effective function is obtained in the model with suppression of either component of this modulation because the feedback inhibition can be entirely shut down by either type of suppression. Thus with strong 
suppression of excitatory input to interneurons no suppression of inhibitory transmission is necessary, and with strong suppression of inhibitory transmission no suppression of excitatory input is necessary. Intermediate levels of suppression can provide good performance if they are combined, but this requires $>50 \%$ suppression of both parameters. Thus, although decreases in excitatory feedback can be more easily compensated for by suppression of excitatory input to interneurons $\left(W^{\prime}\right)$, the actual associative memory function of the network can be aided by suppression of either component of feedback inhibition. When network function was tested with highly overlapping patterns, such as those illustrated in Fig. 4, the range of effective function was more narrow. As shown in Fig. $6 B$, for low values of suppression of inhibition the performance is low because of insufficient learning of the stored patterns, whereas for high levels of suppression of inhibition the performance is low because of interference between the stored patterns. This demonstrates that effective function is only obtained when modulation of feedback excitation is associated with modulation of feedback inhibition with very specific relative values.

\section{EX P ER IMENT A L D A T A}

\section{Cholinergic modulation of inhibitory synaptic potentials}

The resting membrane potential for the pyramidal neurons was typically approximately $-72 \mathrm{mV}(72.35 \pm 4.1, n=40)$. At this resting membrane potential IPSPs were not always prominent, and the cells were depolarized by injection of a constant DC current to help observation of the inhibitory synaptic potentials. Inhibitory potentials obtained at different membrane potentials during stimulation of layer $\mathrm{Ib}$ are shown in Fig. $7 A$. Depolarization of the neuron membrane potential allowed observation of the early and late components of the IPSPs, as can be seen in Fig. 7A. Laminar differences in the components of the IPSPs were observed, with the early $\mathrm{Cl}^{-}$ component being more prominent during the stimulation of the association fiber layer (layer $\mathrm{Ib}$ ), whereas the late component was observed during stimulation of both the layers. As shown in Fig. 9A, inhibitory synaptic potentials elicited during stimulation of the afferent fiber layer (layer Ia) rarely evoked a prominent early inhibitory component.

IPSPs were more easily isolated by pharmacologically blocking the EPSPs with D-APV and CNQX as shown for Ib stimulation in Fig. $8 A$ and for Ia stimulation in Fig. 9, $A$ and $B$. This blockade reduced the peak amplitude of the excitatory component of potentials by $71.4 \pm 7.98 \%(n=6)$ for afferent layer stimulation and $76 \pm 9.8 \%(n=5)$ for asociation fiber stimulation.

Perfusion of the cholinergic agonist carbachol in the slice chamber caused a significant suppression of inhibitory synaptic potentials elicited by stimulation of association/intrinsic fibers in layer Ib, as shown in Figs. $7 B$ and $8, A$ and $B$. As can be seen in these figures, carbachol caused suppression of both the early and late components of the inhibitory synaptic potential. The strong suppression of the fast IPSP component made quantitative measurements difficult. Measurement of the slow inhibitory synaptic potentials $(n=14)$ recorded with sharp electrode techniques revealed that layer Ib inhibitory synaptic potentials were reduced on average by $79.4 \pm 2.4 \%$. This effect was

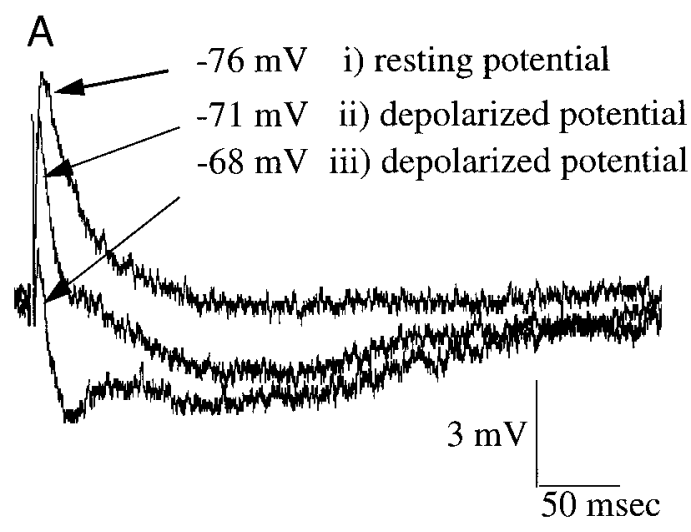

B depolarized potential
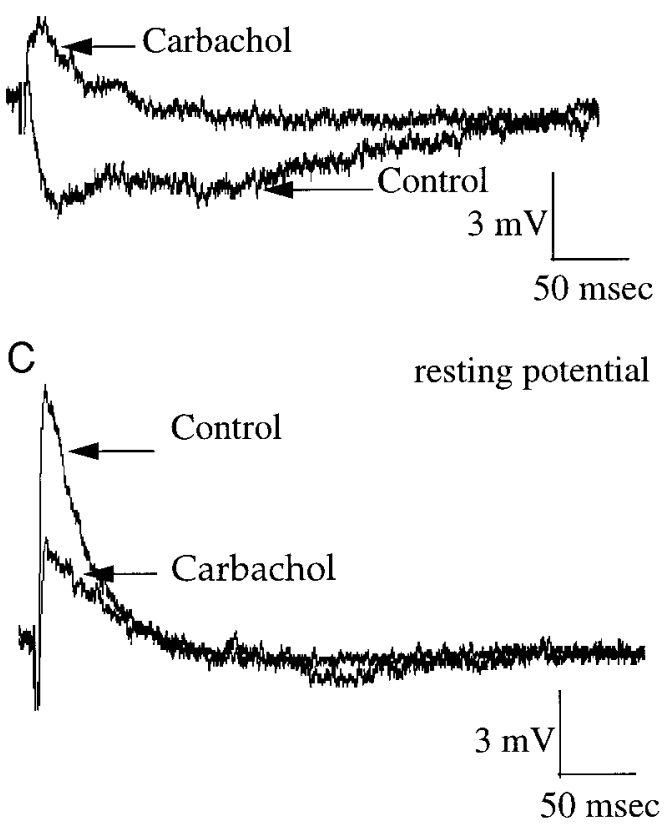

FIG. 7. A: intracellular excitatory and inhibitory synaptic potentials evoked by stimulating the association fiber layer (layer $\mathrm{Ib}$ ). Responses are at resting membrane potential and at different depolarized membrane potentials induced by current injection. Both the slow and fast components of the inhibitory postsynaptic potentials (IPSPs) can be seen in these recordings. The early $\mathrm{Cl}^{-}$ component of the IPSP is more apparent at depolarized potentials. $B$ : effects of bath-applied carbachol exposure are compared in the superimposed traces at depolarized potentials and indicate a suppression of both the fast and slow components of IPSP by the cholinergic agonist. $C$ : perfusion of carbachol also suppressed the excitatory postsynaptic potentials (EPSPs) recorded during stimulation of association/intrinsic fibers in layer $\mathrm{Ib}$ as compared in the overlaid traces at resting membrane potential.

highly statistically significant $(P<0.0007)$. After washing the carbachol out of the slice chamber, the IPSP amplitudes recovered to $\sim 60 \%$ of their control value. This partial recovery may be due to insufficient wash period in some slices because wash takes $>45 \mathrm{~min}$. Experiments with pharmacological blockade of excitatory currents further confirmed this evidence for suppression of inhibitory currents by carbachol. After postsynaptic blockade of excitatory potentials, the decrease in the late evoked Ib inhibitory potentials was found to be $61.75 \pm 4.7 \%$ $(n=5)$. The early evoked Ib inhibitory potentials showed what appeared to be total suppression in carbachol. In carbachol there was no longer a significant difference from baseline at the 
A

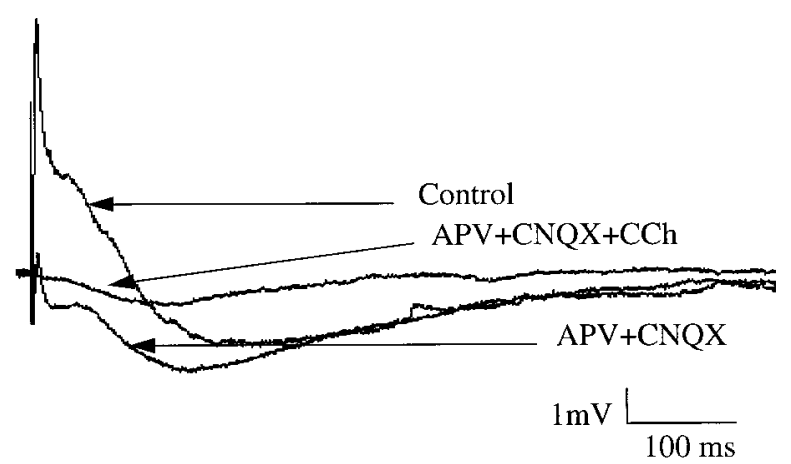

B

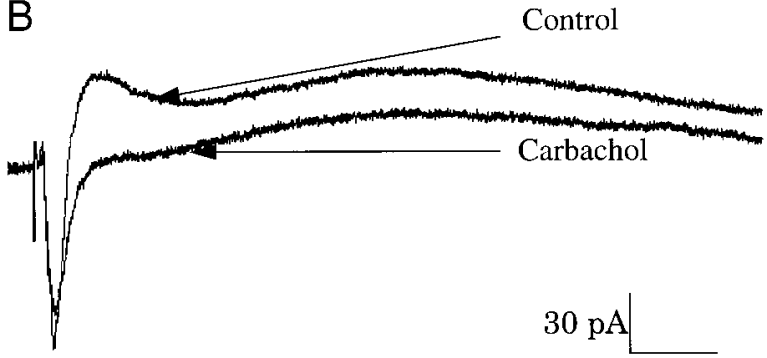

$30 \mathrm{msec}$

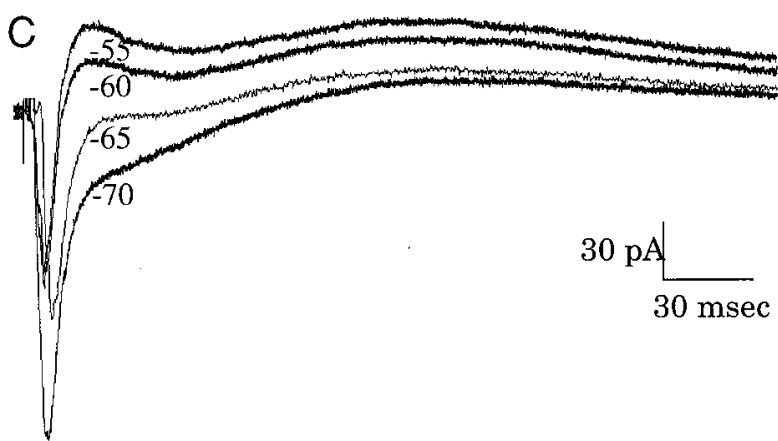

FIG. 8. A: effect of cholinergic modulation on pharmacologically isolated IPSPs evoked by layer Ib stimulation. The EPSP present in the control condition trace (Control) is greatly decreased by bath application of $\mathrm{D}(-)-2-$ amino-5-phosphonovaleric acid and 6-cyano-7-nitroauinoxaline-2,3-dione $(\mathrm{APV}+\mathrm{CNQX})$. During perfusion of carbachol (APV + CNQX + CCh), both the fast and slow components of the IPSP are suppressed. $B$ : cholinergic suppression was also demonstrated in the voltage-clamp mode. Stimulation of layer $\mathrm{Ib}$ when the cell was held at $-60 \mathrm{mV}$ elicited a fast EPSC followed by fast and slow IPSCs (Control). Perfusion of the slice chamber with carbachol suppressed both IPSC components (Carbachol). C: IPSCs evoked at holding potentials between -70 and $-55 \mathrm{mV}$ show the fast and slow inhibitory components peaking between $20-30$ and $130-140 \mathrm{~ms}$ after the stimulus was applied.

time point of the early component of the inhibitory potential (see Fig. 8A).

In comparison, perfusion of carbachol had a weaker effect on inhibitory synaptic potentials elicited by stimulation of afferent fibers in layer Ia, as shown in Fig. 9A. Measurement of the change in IPSPs in a number of slices $(n=16)$ revealed that IPSPs elicited by layer Ia stimulation were decreased on average by $18.5 \pm 3.2 \%$. This effect was just statistically significant $(P<0.05)$. This decrease in the IPSPs caused by carbachol was found to be present in layer Ia when IPSPs were pharmacologically isolated $(19.8 \pm 8, n=6$ ) (see Fig. 9, $A$ and $B)$. The mean effect of carbachol on inhibitory synaptic poten- tials elicited by stimulation in the two layers is summarized in Fig. 10. These results are consistent with the computational model demonstrating that suppression of excitatory transmission between pyramidal cells (layer $\mathrm{Ib}$ ) can be more effectively offset by suppression of feedback inhibition (layer Ib) than by suppression of feedforward inhibition (layer Ia).

Cholinergic effects on synaptic inhibition were also examined by holding membrane potential at $-60 \mathrm{mV}$ in the voltageclamp mode. Figure $8 B$ shows the decrease in the fast and slow IPSCs after exposure to carbachol in the association layer (these currents are shown for different holding potentials in Fig. 8C). An example of the smaller decrease in layer Ia IPSCs is seen in Fig. 9, $A$ and $B$, after the blockade of the EPSCs with $\mathrm{D}-\mathrm{APV}$ and CNQX.

\section{Cholinergic effect on excitatory synaptic potentials}

The effect of carbachol on the height of excitatory synaptic potentials was also analyzed. Carbachol caused a substantial decrease in the height of excitatory synaptic potentials elicited by stimulation of association/intrinsic fibers in layer Ib while having a much weaker effect on the height of excitatory syn-
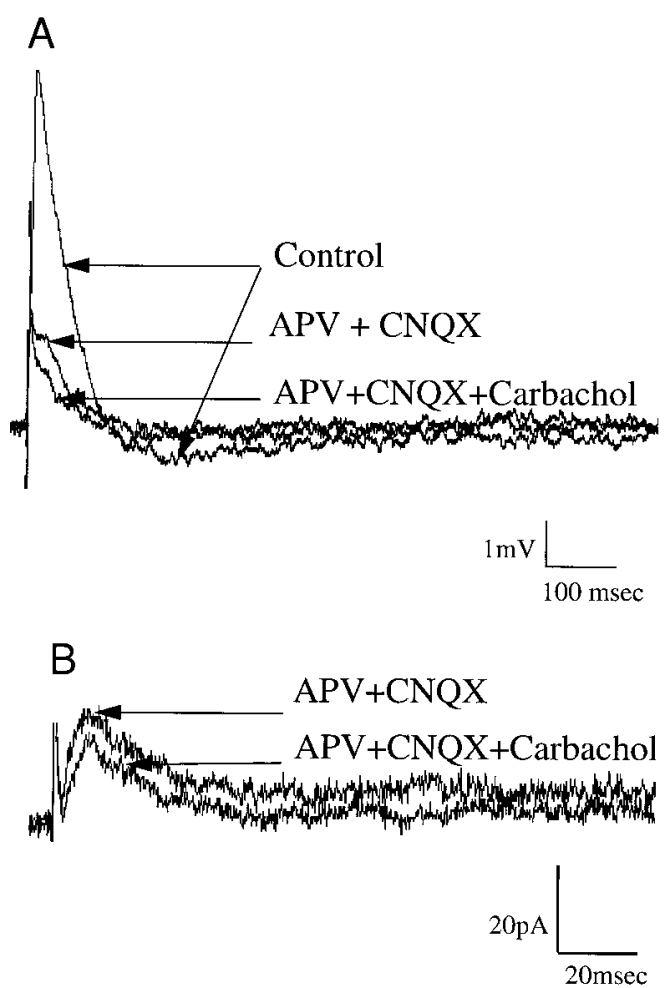

FIG. 9. Intracellular excitatory and inhibitory synaptic potentials evoked by stimulating the afferent fiber layer (layer Ia). The fast IPSP component is not prominent as in the case of inhibitory synaptic potential evoked by layer $\mathrm{Ib}$ stimulation. $A$ : cholinergic effect on isolated IPSP at rest in the afferent layers is shown by the comparison in the overlapping the traces. The excitatory synaptic potential observed in control conditions (Control) is greatly reduced by perfusion of glutamatergic receptor antagonists (CNQX $+\mathrm{APV})$. Carbachol does not dramatically alter the remaining synaptic potentials, illustrating that carbachol-induced suppression of both the inhibitory and excitatory synaptic components is less than that seen with the association layer stimulation. $B$ : cholinergic suppression of the pharmacologically isolated IPSPs in the voltage-clamp mode. The IPSC recorded in the presence of glutamatergic antagonists alone (CNQX + APV) is similar to that observed with the addition of carbachol (CNQX + APV + CCh). Carbachol-induced suppression is not as significant as that in layer $\mathrm{Ib}$. 


\section{$\%$ decrease in layer Ia and Ib evoked EPSPs and IPSPs}

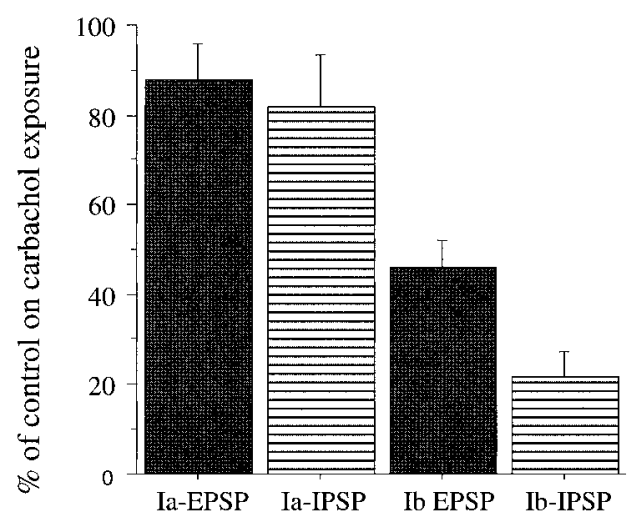

FIG. 10. Histogram comparing the percent suppression of IPSP amplitude (shaded bars) and EPSP amplitudes (solid bars) in the intrinsic/association fiber layer (layer Ib) and in the afferent layer (layer Ia). The suppression of both IPSPs and EPSPs is greater in the association layer than the afferent layer. Also, in the intrinsic/association fiber layer, suppression of inhibitory potentials is greater than that of the excitatory potentials.

aptic potentials elicited in layer Ia. This effect was demonstrated previously with both intracellular and extracellular recording (Hasselmo and Bower 1992). Comparison of EPSPs was documented at resting membrane potentials. The effect on layer Ib EPSPs can be seen in Fig. 7C. Measurement of the change in height of EPSPs elicited by layer Ib stimulation in a number of slices $(n=17)$ demonstrated that carbachol caused a decrease in EPSP height by an average value of $54.1 \pm$ $6.08 \%$. This effect was statistically significant $(P<0.0001)$. EPSPs recovered to $\sim 75 \%$ of their control value when carbachol was washed from the slice. There may be some interaction between the decrease in the amplitude of EPSPs and the fast component of the inhibitory potentials. However, across the population of individual cells, as seen in Fig. 11A, the amount of decrease in layer Ib IPSPs did not seem to be directly related to the EPSP decrease, suggesting that these measurements show effects on two different physiological components of transmission, not just an increase in EPSP potentials because of decreased inhibitory currents. As shown in Fig. 10, the mean effect on inhibitory synaptic potentials elicited by layer Ib stimulation was stronger than the mean effect on excitatory synaptic potentials elicited by layer Ib stimulation.

As demonstrated in a set of previous intracellular recordings, the decrease in EPSPs elicited by stimulation of afferent fibers (in layer Ia) was much smaller than the decrease in EPSPs elicited by layer Ib stimulation. Across a number of slices $(n=$ 18), perfusion of carbachol caused a decrease with an average value of $13.5 \pm 7.78 \%$. This was still statistically significant $(P<0.03)$. As in the associational layer, there seemed no obvious relation between the decrease in the afferent IPSPs and EPSPs, as seen in Fig. $11 B$.

Orthodromic firing threshold decreases in afferent layer and increases in the association layer

The functional advantage of having stronger suppression of excitatory synaptic transmission in layer Ib compared with layer Ia is that it provides a mechanism by which cholinergic modulation could allow afferent input to dominate during storage of new information (Hasselmo et al. 1992). Therefore, we were interested to directly test whether perfusion of the cholinergic agonist carbachol makes it easier for afferent input (layer Ia stimulation) to cause pyramidal cells to spike while making it more difficult for intrinsic input (layer Ib stimulation) to cause pyramidal cells to spike. To test this effect, we obtained strength-duration threshold curves for both layers in control conditions and during cholinergic modulation.

Perfusion of carbachol caused pyramidal neurons to generate action potentials more easily in response to stimulation of afferent input (layer Ia) than in response to stimulation of intrinsic input (layer Ib). Figure 12 shows the threshold stimulus strength-duration curves for a pyramidal neuron during Ia and Ib orthodromic stimulation. Carbachol exposure decreased the firing threshold for stimulation of the afferent fiber layer (layer Ia) in 7 of 10 cells, whereas threshold increased in 3 cells. For the averages and percent changes presented here, the strength of the stimulus at $0.5 \mathrm{~ms}$ was used for the calculations. The average decrease in threshold for layer Ia stimulation across the 10 cells was $28.2 \pm 5.2 \%$. In contrast, the firing threshold for stimulation of the intrinsic fiber layer (layer Ib)
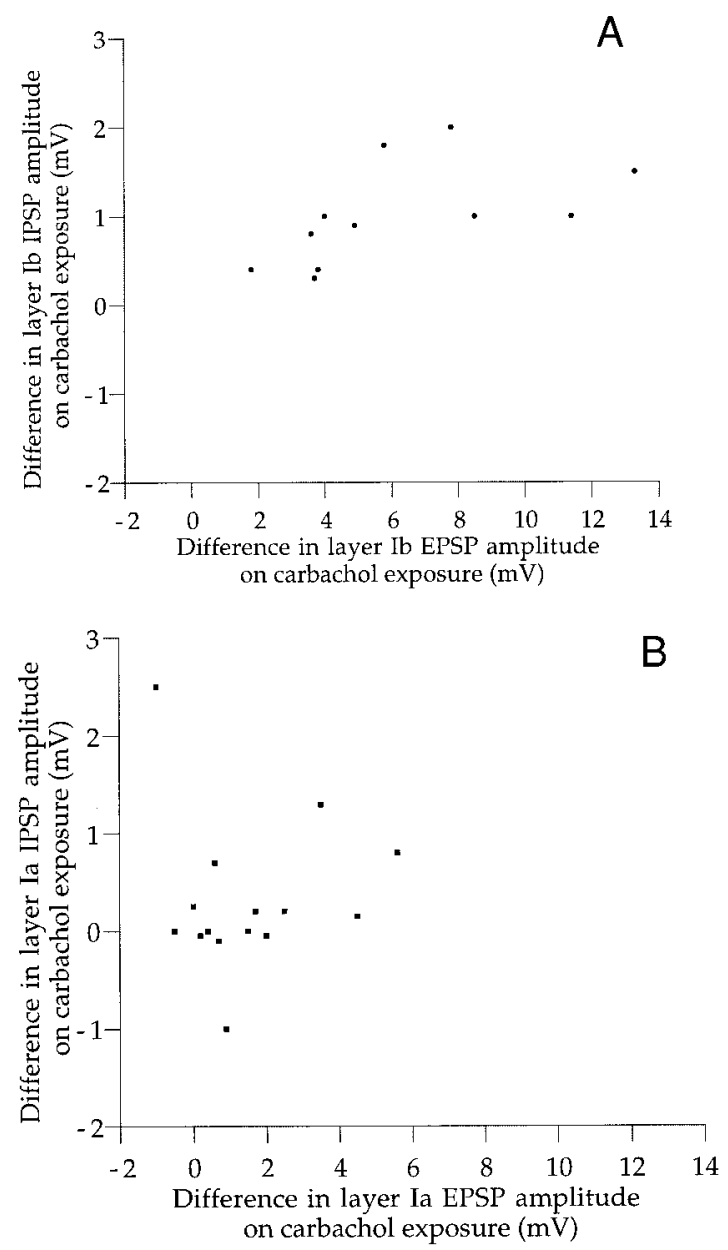

FIG. 11. Relation between the suppression of IPSP and EPSP amplitudes caused by carbachol exposure; the amount of decrease in the excitatory amplitude of each neuron is plotted vs. the corresponding decrease in the inhibitory amplitude of the same neuron. $A$ : across the population of neurons, no obvious relation was noted in the carbachol-induced decrease in excitatory and inhibitory synaptic potentials evoked by stimulating layer Ib. $B$ : decreases in layer Ia-evoked IPSPs and EPSPs also do not appear to be related. 

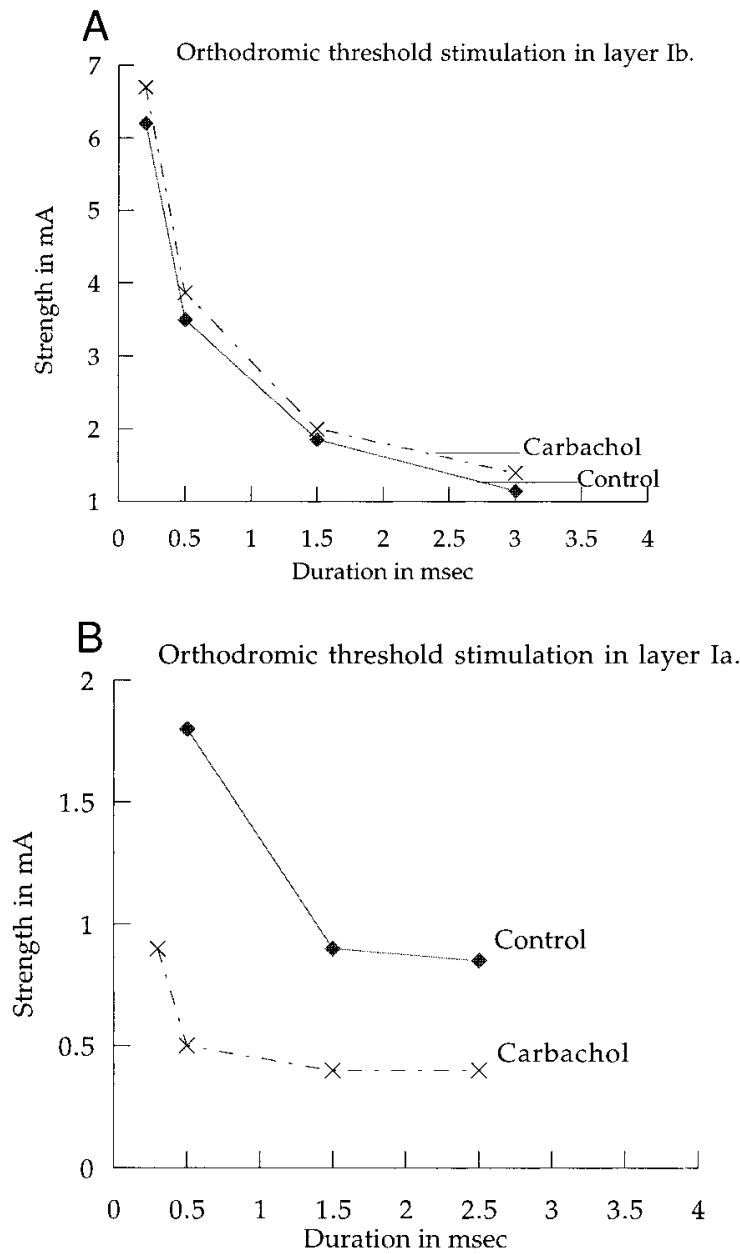

FIG. 12. Strength-duration curves from a neuron showing the difference in the orthodromic firing threshold before and after the introduction of carbachol. These plots show the minimum (threshold) stimulus current strength (in mA) necessary to evoke an intracellularly recorded action potential for different durations of stimulation (in ms). — : minimum values that evoked action potentials in control conditions (before perfusion); - - - : threshold values that evoked action potentials during perfusion of carbachol. A: carbachol causes a slight increase in the threshold for eliciting an action potential with stimulation in the association fiber (layer Ib). $B$ : in contrast, carbachol causes a strong decrease in the threshold for eliciting an action potential with stimulation in the afferent fiber layer (layer Ia).

increased by an average $23.6 \pm 4.6 \%$ in six cells. This differential effect appeared despite the common postsynaptic depolarization of membrane potential during perfusion of carbachol, which alone should make neurons more responsive to both types of stimulation. In these experiments, carbachol depolarized the membrane potential by $6.37 \pm 2.53 \mathrm{mV}(n=$ $15)$ and increased input resistance from $28.2 \pm 8.14 \mathrm{M} \Omega$ to $36.7 \pm 4.03 \mathrm{M} \Omega$, an increase of $\sim 29.6 \pm 4.57 \%,(n=16)$. As demonstrated in previous studies (Barkai and Hasselmo 1994), perfusion of carbachol also decreased the adaptation of pyramidal cells.

\section{I S C U S S I O N}

The experimental data presented here concur with the requirement of the computational model that cholinergic modulation should more strongly suppress inhibitory synaptic potentials evoked by stimulation of layer Ib than inhibitory synaptic potentials evoked by stimulation of layer Ia. IPSPs and IPSCs evoked by stimulation of layer Ib were strongly suppressed during perfusion of the cholinergic agonist carbachol, whereas IPSPs and IPSCs evoked by stimulation of layer Ia were less strongly suppressed. Although cholinergic suppression of evoked IPSPs and IPSCs has been shown in the hippocampus (Haas 1982; Pitler and Alger 1992), this effect was not demonstrated in piriform cortex nor was laminar selectivity of the suppression described previously.

\section{Implications of the model}

The computational modeling presented here demonstrates the requirement that activation of cholinergic receptors should have a stronger effect on intrinsic inhibitory potentials than those activated by stimulation of the afferent fibers. This prediction arose from the combination of previous physiological data showing greater cholinergic suppression of excitatory synaptic transmission in layer Ib than in layer Ia (Hasselmo and Bower 1992) and assumptions about the function of excitatory intrinsic connections within the piriform cortex. Previous modeling demonstrated that cholinergic suppression of excitatory intrinsic connections may be very important for preventing retrieval of previously stored representations from interfering with the storage of new representations (Hasselmo and Schnell 1994; Hasselmo et al. 1992, 1995). Given the possible functional necessity of the suppression of excitatory transmission, the network may then need to compensate for the changes in excitatory transmission through modulation of inhibitory effects.

In our mathematical analysis, we utilized equations for the interaction of excitatory and inhibitory neurons such as those analyzed in previous work (Hasselmo et al. 1995; Pinto et al. 1996; Wilson and Cowan 1972, 1973). In contrast to many abstract mathematical representations of cortical function, these equations have the advantage of explicitly representing separate populations of excitatory and inhibitory neurons, allowing direct analysis of modulatory effects on inhibition. This will be useful for analyzing a wide range of modulatory effects, allowing functional interpretation of a range of specific effects on intrinsic properties as well as synaptic excitation and inhibition within cortical structures. In the mathematical analysis, we made the assumption that equilibrium states of the network should be approximately equal with and without cholinergic modulation. This would assist in associative memory function, allowing the network to have activity patterns of approximately equal amplitude during both encoding and retrieval, aiding in the consistent processing of these patterns by subsequent structures. Some changes in activity were noted during in vivo recording with cholinergic modulation (Beidenbach 1966; Metherate et al. 1990; Sillito and Kemp 1983). However, those experimental data suggest that neuronal activity is stronger during cholinergic modulation, which would require that effects on inhibition should overcompensate for the effects on excitatory transmission (although the cholinergic depolarization of neurons and suppression of adaptation adaptation can also contribute to this increased activity). The analysis presented here is qualitatively similar even if modulation of inhibition overcompensates for the modulation of excitatory transmission.

In addition to the prediction that modulation of feedforward inhibition is less effective at compensating for reduced excitatory feedback, the model suggests differences in the effective- 
ness of different components of feedback inhibition in compensating for reduced excitatory feedback. The suppression of inhibitory synaptic transmission $H$ (i.e., release of GABA) can only partly compensate for suppression of excitatory transmission, whereas the suppression of excitatory input to inhibitory interneurons $W^{\prime}$ (i.e., glutamatergic connections from pyramidal cells to interneurons) more effectively compensates for reduction in excitatory feedback across a wider range of values. This suggests that the cholinergic suppression of inhibitory synaptic potentials evoked by layer Ib stimulation may be more dependent on reduced excitatory input to inhibitory interneurons than on reduced release of the inhibitory transmitter GABA from interneurons. However, some aspects of our data suggest that the effect is not purely due to decreased excitatory input to interneurons, including 1 ) carbachol still suppressed inhibitory potentials elicited during pharmacological blockade of excitatory currents, 2) the effects on inhibitory potentials often appeared sooner than the effect on excitatory potentials, and 3) our data show independence of effects on excitatory and inhibitory potentials. Further experimental data will be necessary to separately analyze cholinergic effects on excitatory input to interneurons $\left(W^{\prime}\right)$ and the release of GABA from interneurons $(H)$ in the piriform cortex.

The network simulations of the piriform cortex allow analysis of the cholinergic modulation of inhibition in the more specific functional framework of attractor dynamics and associative memory function. This work continues previous work exploring the storage of patterns of activity in a network with separate populations of excitatory and inhibitory neurons (Hasselmo and Linster 1998b; Hasselmo et al. 1995, 1997). This model draws on the assumption that the excitatory recurrent connections of the piriform cortex mediate associative memory function, allowing storage of patterns of activity representing odors, and retrieval of these patterns given incomplete pattern cues (Bower 1995; Haberly 1985; Haberly and Bower 1989; Hasselmo and Linster 1998a; Hasselmo et al. 1992; Wilson and Bower 1988). The distinct modeling of separate populations of excitatory and inhibitory neurons used in the model presented here allows detailed analysis of how modulation of inhibition could play a role in setting appropriate functional dynamics in associative memory networks. The exploration of parameter values shown here in Figs. 5 and 6 demonstrates that strong cholinergic suppression of feedback inhibition is necessary for effective function, whereas effective function is obtained at a range of parameters of feedforward inhibition. In fact, previous published versions of this simulation used selective suppression of feedback but not feedforward inhibition (Hasselmo et al. 1995). Models of the olfactory bulb also demonstrated how modulatory effects on inhibition could play a role in setting appropriate dynamics for separation and enhancement of odor responses (Linster and Hasselmo 1997).

More abstract associative memory models assume network activity is clamped to the input pattern during learning (Amit 1988). In this model, the internally regulated cholinergic suppression of excitatory feedback combined with suppression of feedback inhibition allows the network to preferentially respond more to afferent input during encoding. In our experimental data, cholinergic modulation also appears to make afferent input the predominant influence on neuronal activity. As shown in the strength-duration curves in Fig. 12, the threshold for eliciting an action potential with afferent fiber stimulation (layer Ia) is strongly decreased in the presence of carbachol (because of the direct depolarization of pyramidal cell membrane potential), whereas the threshold for eliciting an action potential with intrinsic fiber stimulation (layer Ib) is increased (because of the suppression of excitatory intrinsic synaptic transmission). These results support the modeling proposal that cholinergic modulation allows afferent input to dominate without greatly changing the net activity within the network.

Whereas these models assume the importance of stable attractor dynamics in the network, alternative interpretations are possible. For example, one model of piriform cortex proposes that sequential cycles of activity allow hierarchical classification of odor information (Ambros-Ingerson et al. 1990; Granger et al. 1989). These models are not entirely inconsistent with the one presented here, in that the afferent connections in the model presented here could undergo self-organization to form categories such as those in the Ambros-Ingerson model. The relative strength of inhibition and excitation is very important for the function of that previous model as well. In particular, strong excitatory feedback connections could interfere with effective formation of categories; therefore it might be important to suppress feedback. At the same time, suppression of inhibition would become important to allow sufficient activity to underlie formation of new categories. This is consistent with evidence that long-term potentiation occurs in the piriform cortex during cholinergic modulation (Hasselmo and Barkai 1995; Patil et al. 1998) and in behavioral contexts requiring learning (Roman et al. 1987, 1993a,b).

Other functional interpretations of piriform cortex activity have been developed. For example, the representation of odors was described in terms of limit cycles or chaotic attractors in modeling and electroencephalogram work by Freeman and others (Freeman 1975; Freeman et al. 1988; Liljenstrom and Hasselmo 1995; Liljenstrom and Wu 1995; Yao and Freeman 1990). These representations are considerably more complex than the fixed point attractors shown here, but similar principles apply. If a particular dynamical state must represent a particular odor stimulus during two very different arousal states (with different levels of cholinergic modulation), each cholinergic effect on a parameter of cortical function must be compensated for such that the dynamical state has a consistent and recognizable influence on other cortical regions. Otherwise, the reduced feedback excitation would cause a very different dynamical pattern of activity for the same sensory stimulus during different modulatory states.

The fixed point attractors used here do not differ dramatically in their properties from limit cycle attractors used in other models, but limit cycle attractors or sequences of neuronal activity are probably a more realistic neuronal representation. Here we focused on isolated storage and retrieval of single patterns, but the real network must deal with an ongoing interaction with continuously changing olfactory input and behavioral contingencies. It is very likely that crosstemporal interitem associations are stored and retrieved in this context. In fact, unit recording in piriform cortex during performance of an olfactory discrimination task demonstrates activity to multiple task components, not just odor sampling (Schoenbaum and Eichenbaum 1995). The compensation of overall activity would still be relevant to storage of sequences, but there may be additional dynamical properties of these influences that will become clearer with more detailed functional models. 


\section{Relation to previous physiological data}

Our experimental data show that inhibitory synaptic potentials evoked by stimulation of the piriform cortex are suppressed by cholinergic modulation in a manner similar to the cholinergic suppression of evoked inhibitory synaptic potentials in the hippocampus (Haas 1982; Pitler and Alger 1992). The modulation of inhibition could be important for offsetting a loss of excitatory transmission in the hippocampus as well because cholinergic modulation has been shown to suppress excitatory synaptic potentials in region $\mathrm{CA} 3$ and region $\mathrm{CA} 1$ of the hippocampus (Hasselmo and Schnell 1994; Hasselmo et al. 1995; Hounsgaard 1978; Valentino and Dingledine 1981). Most of the cholinergic effects on inhibitory potentials and currents shown here could be due to either a decrease in the excitatory synaptic input to interneurons or in the inhibitory transmission from interneurons. However, this study also shows that evoked inhibitory synaptic potentials and currents recorded in the presence of CNQX and APV are suppressed during perfusion of carbachol. This is consistent with previous studies in hippocampus showing suppression of monosynaptic evoked inhibitory currents (Pitler and Alger 1992) and decreased frequency of TTX-insensitive spontaneous inhibitory currents (Behrends and ten Bruggencate 1993). Suppression of inhibitory transmission was also demonstrated in cultures of neocortical neurons (Kimura and Baughman 1997). This suggests that the reduction in evoked inhibitory synaptic potentials is not just due to a reduction in the excitatory input to interneurons.

Here we show cholinergic suppression of both the fast and slow components of inhibitory synaptic potentials. Previous work on inhibitory synaptic potentials in the hippocampus reported an effect on both the fast and slow components (Pitler and Alger 1992), although the bulk of previously presented data concerned the fast $\mathrm{GABA}_{\mathrm{A}}$-mediated inhibitory potentials. Other studies analyzed cholinergic modulation of fast and slow inhibitory potentials in a more detailed manner (Muller and Misgeld 1989). In our data, the fast inhibitory synaptic potentials are much more prominent with stimulation of layer Ib than of layer Ia. Previous work in the piriform cortex has repeatedly shown both components after layer $\mathrm{Ib}$ stimulation (Tseng and Haberly 1989). Our data suggest that stimulation in the afferent fiber layer predominantly evokes slower components of the IPSP. This is consistent with more recent data showing a greater proportion of slow GABA currents in more distal dendritic regions (Kapur et al. 1997). If there is a laminar difference in the relative amount of fast and slow inhibitory potentials evoked by stimulation, this would be consistent with data from hippocampal region CA1, where it has been shown that stimulation of feedforward inhibition in stratum lacunosum-moleculare selectively activates slower components of inhibition (Lacaille and Schwartzkroin 1988).

Cholinergic agonists have been shown to increase frequency of spontaneous inhibitory potentials recorded intracellularly from pyramidal cells in brain slice preparations of the hippocampus (Pitler and Alger 1992). This effect was also observed in the piriform cortex in the presence of a range of modulatory substances (Gellman and Aghajanian 1993), including the cholinergic agonist carbachol (R. Gollub, personal communication). This increase in spontaneous inhibitory potentials was attributed to a direct depolarization of inhibitory interneurons caused by activation of cholinergic receptors (Behrends and ten Bruggencate 1993; Pitler and Alger 1992), which was demonstrated with intracellular recording from interneurons in the hippocampus (McQuiston and Madison 1996; Reece and Schwartzkroin 1991). This effect appears somewhat paradoxical with relation to the observed suppression of evoked inhibitory potentials but may be due to a requirement for lower tonic background activity, with greater response to specific evoked patterns of activity. This could contribute to a change in "signal-to-noise ratio" similar to that proposed for effects of noradrenergic modulation. As shown in Fig. 3, depolarized interneurons will result in an overall increase of inhibitory tone, decreasing background activity, whereas suppression of excitatory input to interneurons will results in less feedback inhibition during activity elicited by afferent input.

The authors thank Dr. Donald Rannie for help with voltage-clamp recordings.

This research was supported by National Institute of Mental Health Grant R29 MH-52732.

Address for reprint requests: M. E. Hasselmo, Dept. of Psychology, Boston University, 64 Cummington St., Boston, MA 02215.

Received 10 September 1997; accepted in final form 6 January 1999.

\section{REFERENCES}

Ambros-Ingerson, J., Granger, R., AND LynCH, G. Simulation of paleocortex performs heirarchical clustering. Science 247: 1344-1348, 1990.

Amit, D. J. Modeling Brain Function: The World of Attractor Neural Networks. Cambridge, UK: Cambridge University Press, 1988.

Barkai, E., Bergman, R. E., Horwitz, G., And Hasselmo, M. E. Modulation of associative memory function in a biophysical simulation of rat piriform cortex. J. Neurophysiol. 72: 659-677, 1994.

BARKAI, E. AND HASSElmo, M. E. Modulation of the input/output function of rat piriform cortex pyramidal cells. J. Neurophysiol. 72: 644-658, 1994.

Behrends, J. C. AND TEN BRuggencate, G. Cholinergic modulation of synaptic inhibition in the guinea pig hippocampus in vitro: excitation of GABAergic interneurons and inhibition of GABA-release. J. Neurophysiol. 69: 626-629, 1993.

BeIDENBACH, M. A. Effects of anaesthetics and cholinergic drugs on prepyriform electrical activity in cats. Exp. Neurol. 16: 464-479, 1966.

BOWER, J. M. Reverse engineering the nervous system: an in vivo, in vitro and computational approach to understanding the mammalian olfactory system. In: An Introduction to Neural and Electronic Networks, edited by S. Zornetzer, J. Davis, and C. Lau. New York: Academic, 1995.

Burgard, E. C. And SARvey, J. M. Muscarinic receptor activation facilitates the induction of long-term potentiation (LTP) in the rat dentate gyrus. Neurosci. Lett. 116: 34-39, 1990.

FRANSEN, E. AND LANSNER, A. Low spiking rates in a population of mutually exciting pyramidal cells, Network 6: 271-288, 1995.

Freeman, W. J. Mass Action in the Nervous System. New York: Academic, 1975.

Freeman, W. J., Yao, Y., And Burke, B. Central pattern generating and recognizing in olfactory bulb: a correlation learning rule. Neural Networks 1: 277-288, 1988.

Gellman, R. L. And Aghajanian, G. K. Pyramidal cells in piriform cortex receive a convergence of inputs from monoamine activated GABAergic interneurons. Brain Res. 600: 63-73, 1993.

Granger, R., Ambros-Ingerson, J., And Lynch, G. Derivation of encoding characteristics of layer II cerebral cortex. J. Cogn. Neurosci. 1: 61-87, 1989.

HAAs, H. L. Cholinergic disinhibition in hippocampal slices of the rat. Brain Res. 233: 200-204, 1982.

HABERLY, L. B. Neuronal circuitry in olfactory cortex: anatomy and functional implications. Chem. Senses. 10: 219-238, 1985.

HABERLY, L. B. AND Bower, J. M. Olfactory cortex: model circuit for study of associative memory? Trends Neurosci. 12: 258-264, 1989.

HANSEL D. AND SOMPOLINSKY H. Modeling feature selectivity in local cortical circuits. In: Methods in Neuronal Modeling, edited by C. Koch and I. Segev. Cambridge, MA: MIT Press, 1998.

Hasselmo, M. E. Acetylcholine and learning in a cortical associative memory. Neural Comput. 5: 32-44, 1993. 
Hasselmo, M. E. Neuromodulation and cortical function: modeling the physiological basis of behavior. Behav. Brain Res. 67: 1-27, 1995.

Hasselmo, M. E., ANDerson, B. P., AND Bower, J. M. Cholinergic modulation of cortical associative memory function. J. Neurophysiol. 67: 1230-1246, 1992.

Hasselmo, M. E. AND BARKAI, E. Cholinergic modulation of activity-dependent synaptic plasticity in the piriform cortex: brain slice physiology and computational modeling. J. Neurosci. 15: 6592-6604, 1995.

Hasselmo, M. E. and Bower, J. M. Cholinergic suppression specific to intrinsic not afferent fiber synapses in rat piriform (olfactory) cortex. J. Neurophysiol. 67: 1222-1229, 1992.

Hasselmo, M. E. And Bower, J. M. Acetylcholine and memory. Trends Neurosci. 16: 218-222, 1993.

Hasselmo, M. E. AND CeKic, M. Suppression of synaptic transmission allows combination of associative feedback and self-organizing feedforward connections in a model of neocortex. Behav. Brain Res. 79: 153-161, 1996.

Hasselmo, M. E. and Linster, C. Modeling the piriform cortex. In: Models of Cortical Circuits. Cerebral Cortex, edited by E. G. Jones, P. S. Ulinski, and A. Peters. New York: Plenum, 1998a, vol. 13.

Hasselmo, M. E. AND Linster, C. Acetylcholine and frontal cortex "signalto-noise" ratio. In: The Human Frontal Lobes: Function and Disorders, edited by B. L. Miller and J. Cummings. New York: Guilford, 1998b.

Hasselmo, M. E., Linster, C., Ma, D., And Cekic, M. Noradrenergic suppression of synaptic transmission may influence cortical "signal-to-noise" ratio. J. Neurophysiol. 77: 3326-3339, 1997.

Hasselmo, M. E. AND Schnell, E. Laminar selectivity of the cholinergic suppression of synaptic transmission in rat hippocampal region CA1: computational modeling and brain slice physiology. J. Neurosci. 14: 3898-3914, 1994.

Hasselmo, M. E., Schnell, E., and Barkai, E. Dynamics of learning and recall at excitatory recurrent synapses and cholinergic modulation in hippocampal region CA3. J. Neurosci. 15: 5249-5262, 1995.

HOUNSGAARD, J. Presynaptic inhibitory action of acetylcholine in area CA1 of the hippocampus. Exp. Neurol. 62: 787-797, 1978.

Huerta, P. T. AND Lisman, J. E. Heightened synaptic plasticity of hippocampal CA1 neurons during a cholinergically induced rhythmic state. Nature 364: 723-725, 1993.

Kapur, A., Pearce, R. A., Lytton, W. W., and Haberly, L. B. GABA A $^{-}$ mediated IPSCs in piriform cortex have fast and slow components with different properties and locations on pyramidal cells. J Neurophysiol. 78: 2531-2545, 1997.

Kimura, F. And Baughman, R. W. Distinct muscarinic receptor subtypes suppress excitatory and inhibitory synaptic responses in cortical neurons. J. Neurophysiol. 77: 709-716, 1997.

LACAILLE, J. C. AND SChwARTZKROIN, P. A. Stratum lacunosum-moleculare interneurons of hippocampal CA1 region. I. Intracellular response characteristics, synaptic responses and morphology. J. Neurosci. 8: 1400-1410, 1988.

Levy, W. B., Colbert, C. M., and Desmond, N. L. Elemental adaptive processes of neurons and synapses: a statistical/computational perspective. In: Neuroscience and Connectionist Theory, edited by M. A. Gluck and D. E. Rumelhart. Hillsdale, NJ: Erlbaum, 1990, p. 187-236.

Liljenstrom, H. and Hasselmo, M. E. Cholinergic modulation of cortical oscillatory dynamics. J. Neurophysiol. 74: 288-297, 1995.

LiLJENSTROM, H. AND Wu, X. B. Noise-enhanced performance in a cortical associative memory model. Int. J. Neural Syst. 6: 19-29, 1995.

Linster, C. AND Hasselmo, M. E. Modulation of inhibition in a model of olfactory bulb reduces the overlap in the neural representation of olfactory stimuli. Behav. Brain Res. 84: 117-127, 1997.

Linster, C., Wyble, B. P., and Hasselmo, M. E. Reciprocal interactions between the olfactory system and the horizontal limb of the diagonal band of Broca. Soc. Neur. Abstr. 23: 807.8, 1997.

Linster, C., Wyble, B. P., and Hasselmo, M. E. Modulation of synaptic potentials in the piriform cortex by electrical stimulation of the horizontal limb of the diagonal band of Broca. J. Neurophysiol. In press.
McQuiston, A. R. AND Madison, D. V. Postsynaptic actions of cholinergic receptor activation on multiple types of interneurons in CA1 region of the rat hippocampus. Soc. Neurosci. Abstr. 22: 786, 1996.

Metherate, R., Ashe, J. H., AND Weinberger, N. M. Acetylcholine modifies neuronal acoustic rate-level functions in guinea pig auditory cortex by an action at muscarinic receptors. Synapse 6: 364-368, 1990.

MulleR, W AND Misgeld, U. Carbachol reduces $\mathrm{I}_{\mathrm{Kbaclofen}}$, but not $\mathrm{I}_{\mathrm{KGABA}}$ in guinea pig hippocampal slices. Neurosci. Lett. 102: 229-234, 1989.

Patil, M. M., Linster, C., Lubenov, E., and Hasselmo, M. E. Cholinergic agonist carbachol enables associative long-term potentiation in piriform cortex slices. J. Neurophysiol. 80: 2467-2474, 1998.

Pinto, D. J., Brumberg, J. C., Simons, D. J., and Ermentrout, G. B. A quantitative population model of whisker barrels: re-examining the WilsonCowan equations. J. Comput. Neurosci. 3: 247-264, 1996.

Pitler, T. A. AND Alger, B. E. Cholinergic excitation of GABAergic interneurons in the rat hippocampal slice. J. Physiol. (Lond.). 450: 127-142, 1992.

Reece, L. J. And Schwartzkroin, P. A. Effects of cholinergic agonists on 2 nonpyramidal cell-types in rat hippocampal slices. Brain Res. 566: 115-126, 1991.

Roman, F., Staubli, U., And Lynch, G. Evidence for synaptic potentiation in a cortical network during learning. Brain Res. 418: 221-226, 1987.

Roman, F. S., Simonetto, I., AND SoumiReu-Mourat, B. Learning and memory of odor-reward association: selective impairment following horizontal diagonal band lesions. Behav. Neurosci. 107: 72-81, 1993.

Roman, F. S., Chaillan, F. A., And Soumireu-Mourat, B. Long-term potentiation in rat piriform cortex following discrimination learning. Brain Res. 601: 265-72, 1993.

Schoenbaum, G. and Eichenbaum, H. Information coding in the rodent prefrontal cortex. I. Single-neuron activity in orbitofrontal cortex compared with that in pyriform cortex. J Neurophysiol. 74: 733-750, 1995.

Sillito, A. M. AND KemP, J. A. Cholinergic modulation of the functional organization of the cat visual cortex. Brain Res. 289: 143-155, 1983.

TSENG, G.-F. AND HaBERLY, L. B. Characterization of synaptically mediated fast and slow inhibitory processes in piriform cortex in an in vitro slice preparation. J. Neurophysiol. 59: 1352-1376, 1988.

Tsodyks, M. V., Skaggs ,W. E., Sejnowski, T. J., and McNaughton, B. L. Paradoxical effects of external modulation of inhibitory interneurons. J. Neurosci. 17: 4382-4388, 1997.

Valentino, R. J. And Dingledine, R. Presynaptic inhibitory effect of acetylcholine in the hippocampus. J. Neurosci. 1: 784-792, 1981.

Williams, S. H. And Constanti, A. A. Quantitative study of the effects of some muscarinic antagonists on the guinea-pig olfactory cortex slice. $\mathrm{Br} . \mathrm{J}$. Pharmacol. 93: 855-862, 1988.

WILSON, H. R. AND CowAN, J. D. Excitatory and inhibitory interactions in localized populations of model neurons. Biophys. J. 12: 1-24, 1972.

Wilson, H. R. AND Cowan, J. D. A mathematical theory of the functional dynamics of cortical and thalamic nervous tissue. Kybernetik 13: 55-80, 1973.

Wilson, M. A. AND Bower, J. M. A computer simulation of olfactory cortex with functional implications for storage and retrieval of olfactory information. In: Advances in Neural Information Processing Systems, edited by D. Amderson. New York: AIP, 1988, p. 114-126.

Yamamoto, C. AND KawaI, N. Presynaptic action of acetylcholine in thin sections from the guinea-pig dentate gyrus in vitro. Exp. Neurol. 19: 176187, 1967.

YaO, Y. AND Freeman, W. J. Model of biological pattern recognition with spatially chaotic dynamics. Neural Networks 3: 153-170, 1990. 